\title{
Surrogate Model-Based Strategy for Cryogenic Cavitation Model Validation and Sensitivity Evaluation
}

\author{
Tushar Goel $^{* 1}$, Jinhui Zhao ${ }^{\dagger 2}$, Siddharth Thakur ${ }^{\ddagger 1}$, Raphael T. Haftka ${ }^{\S 1}$, and Wei Shyy ${ }^{* 2}$ \\ ${ }^{I}$ Department of Mechanical and Aerospace Engineering, University of Florida, Gainesville, FL 32611 \\ ${ }^{2}$ Department of Aerospace Engineering, University of Michigan, Ann Arbor, MI 48109
}

\begin{abstract}
Cryogenic cavitation experiences phase change in an environment where the vapor pressure is temperature dependent. The cavitation dynamics have critical implications on the performance and safety of liquid rocket engines, but there is no established method to estimate the actual loads due to cavitation on the inducer blades. To help develop such a computational capability, we conduct a systematic investigation of a transport-based, homogeneous cryogenic cavitation model for code validation and model improvement exercises. We assess the role of model parameters in the cavitation model and uncertainties in material properties via global sensitivity analysis coupled with multiple surrogate models including polynomial response surface, radial basis neural network, Kriging and a weighted average composite model. The results indicate that while the predictions are more sensitive to changes in cavitation model parameters than uncertainties in material properties, the impact of uncertainty in temperature dependent vapor pressure on the performance is significant. We calibrate the cryogenic cavitation model parameters using a multiple surrogates-based optimization strategy. The optimal parameters increase the importance of condensation terms and show improved prediction performance on a number of benchmark problems.
\end{abstract}

b

$C_{\text {dest }}, C_{\text {prod }}$

$C_{p}$

$C_{p m}$

$C_{\varepsilon_{1}}, C_{\varepsilon_{2}}$

$\mathrm{D}$

$\mathrm{E}(f(x))$

$f(\mathbf{x})$

$f_{v}$

$h$

$k$

$L$

$\dot{m}^{-}, \dot{m}^{+}$

$\min (a, b)$

$\max (\mathrm{a}, \mathrm{b})$

$N_{R B F}$

\section{Nomenclature}

Estimated coefficient vector associated with polynomial basis functions

Empirical parameters used in cavitation model

Pressure coefficient

Specific heat of mixture

$k-\varepsilon$ turbulence model coefficients

Characteristic length scale

Expected value of $f(\mathrm{x})$ with respect to $x$

Function of variable vector $\mathbf{x}$

Mass fraction of vapor

Specific enthalpy

Turbulent kinetic energy

Latent heat of vaporization

Cavitation source terms

Minimum of $a$ and $b$

Maximum of $a$ and $b$

Number of radial basis functions

${ }^{*}$ PhD Candidate, Student Member AIAA

${ }^{\dagger}$ Graduate Student, Student Member AIAA

¥Visiting Professor, AIAA Member

$\S$ Distinguished Professor, Fellow AIAA

** Clarence L "Kelly" Johnson Professor, Fellow AIAA 


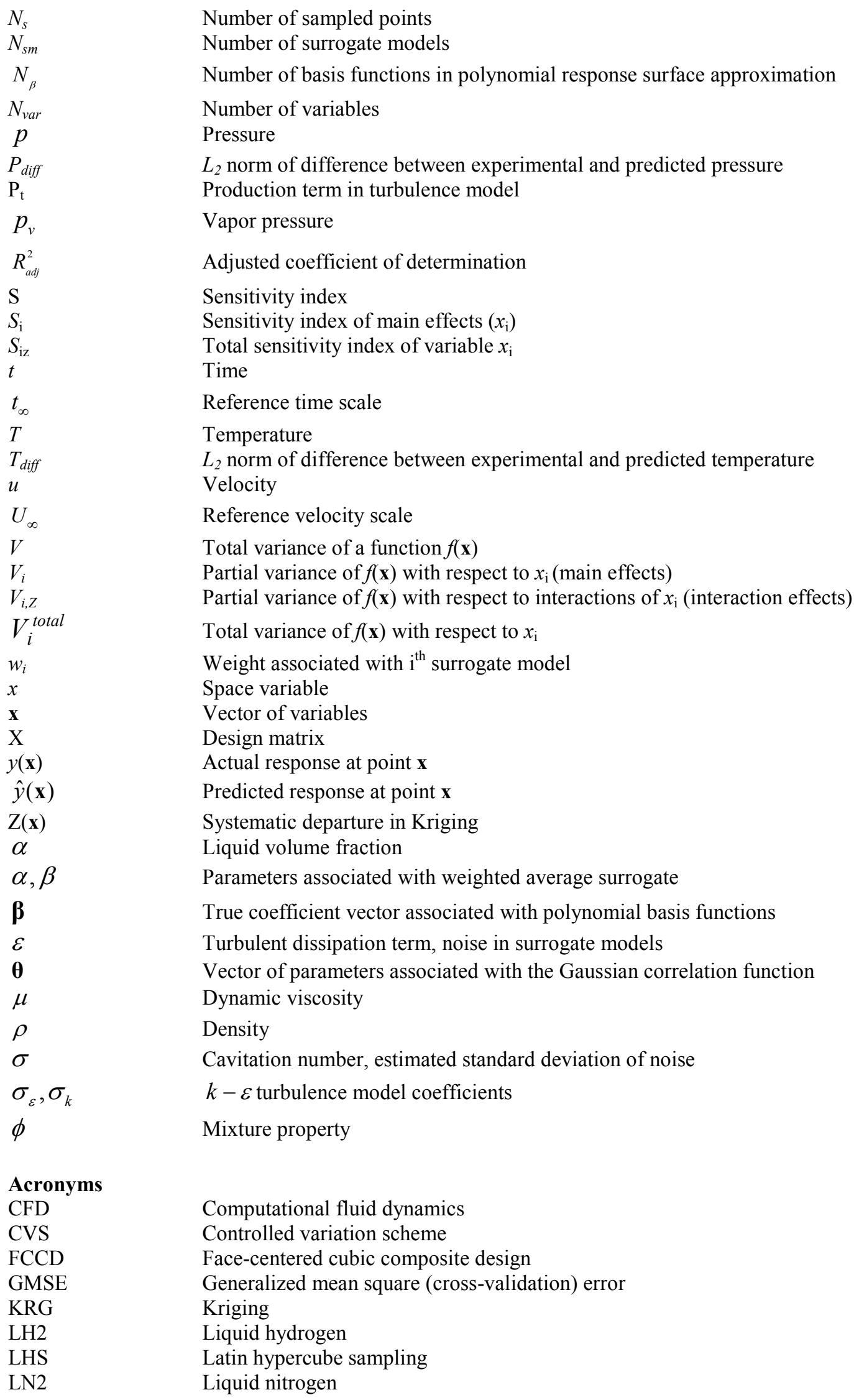




$\begin{array}{ll}\text { LOX } & \text { Liquid oxygen } \\ \text { POF } & \text { Pareto optimal front } \\ \text { PRESS } & \text { Square root of predicted residual sum of squares } \\ \text { PRS } & \text { Polynomial response surface approximation } \\ \text { RBNN } & \text { Radial basis neural network } \\ \text { WTA } & \text { Weighted average surrogate }\end{array}$

$\begin{array}{ll}\begin{array}{l}\text { Subscript } \\ \text { baseline }\end{array} & \text { Baseline parameters } \\ \text { C } & \text { Cavity } \\ 1 & \text { Liquid } \\ \mathrm{m} & \text { Mixture } \\ \mathrm{KRG} & \text { Quantity associated with Kriging } \\ \mathrm{PRS} & \text { Quantity associated with polynomial response surface } \\ \mathrm{RBNN} & \text { Quantity associated with radial basis neural network } \\ \mathrm{rms} & \text { root mean square of the quantity } \\ \mathrm{t} & \text { Turbulent } \\ \mathrm{v} & \text { Vapor } \\ \infty & \text { Free stream quantities }\end{array}$

Superscripts

*

Scale factors and normalized quantities

\section{Introduction}

Cavitation is one of the foremost problems observed in the turbomachinery such as inducers, pumps, turbines, marine propellers, nozzles, hydrofoils etc. due to wide ranging pressure variations in the flow. Cavitation occurs when the local pressure in the flow falls below the vapor pressure and consequently the fluid undergoes a phase change (Batchelor ${ }^{1}$, Brennen $^{2}$ ). Cavitation induces noise, mechanical vibrations, material erosion, and can severely impact the performance as well as the structural integrity of fluid machinery. The study of cavitating flows is complicated by simultaneous presence of turbulence, multiple timescales, large density variations or phase change, interfacial dynamics etc. Due to its practical importance and rich physics, cavitating flow is a topic of significant interest and challenge to the computational community.

The study of cavitating flows in cryogenic environment has practical importance for space applications because cryogens often serve as fuels for the space launch vehicles. For example, a combination of liquid oxygen (LOX) and liquid hydrogen (LH2) is used as rocket propellant mixture because the power/gallon ratio of LH2 is higher compared to other alternatives (NASA Online Facts ${ }^{3}$ ). A key design issue related to such liquid rocket fuel and oxidizer pumps is the minimum pressure that the design can tolerate for a given inlet temperature and rotating speed. To keep inlet pressure low (reduce tank weight) and pump rotational speeds high (reduce engine weight), cavitation is prone to appear in the inducer section. To date, there is no established method to estimate the actual loads due to cavitation on the inducer blades. Virtually every rocket engine system designed in the U.S. has experienced issues with cavitating elements in the pump. This includes recent programs like ATP turbopumps for the SSME, the Fastrac LOX pump, and the RS-68 commercial engine. An integrated framework based on computational modeling and control strategies is desirable to treat this critical and difficult issue. It is clear that the design of efficient turbomachinery components requires understanding and accurate prediction of the cryogenic cavitating flows.

Cavitating flow computations have been conducted using both density-based ${ }^{4-7}$ and pressure-based numerical approaches ${ }^{8-11}$, with the cavitation models developed based on: (i) Rayleigh-Plesset type of bubble formulation $^{12}$ which separates the liquid and vapor region based on the force balance notion, and (ii) homogeneous fluid approach ${ }^{9}$ which treats the cavity as a region consisting of continuous mixture of liquid and vapor phases. In the homogeneous fluid model, the density field is commonly modeled via either a generalized equation of $\operatorname{state}^{13,14}$ or a transport equation of the liquid/vapor phase fraction ${ }^{4,7,9,10,15}$. Recent efforts made in computational and modeling aspects of cavitating flows are discussed by Wang et al. ${ }^{16}$, Senocak and Shyy ${ }^{10,11,17}$, Ahuja et al. ${ }^{5}$, Venkateswaran et al. ${ }^{6}$, Preston et al. ${ }^{18}$, and Utturkar et al. ${ }^{19}$. 
To date, the majority of the cavitation modeling efforts have focused on the assumption that cavitation occurs with negligible energy interactions (isothermal condition). This assumption is reasonable for cavitation in non-cryogenic fluids but fails for thermo-sensible fluids like liquid hydrogen and liquid oxygen (cryogens) due to the differences in material properties (low liquid-vapor density ratio, low thermal conductivities, steep slope of pressure-temperature saturation curves etc.), and the coupling of thermal effects such as variation in vapor pressure/density with temperature etc. (Utturkar et al. ${ }^{15,}{ }^{19}$, Utturkar ${ }^{20}$, Ahuja and Hosangadi $^{21}$ ). Figure $1^{22}$ illustrates the behavior of the physical properties of a representative cryogen, liquid nitrogen, in the liquid-vapor saturation regime. The temperature range in the plots is chosen based on the general operating condition of the fluid, which is close to the critical point for liquid nitrogen (cryogens in general). We observe substantial variation in the material properties with changes in the temperatures.

As discussed by Utturkar et al. ${ }^{19}$, dynamic similarity in case of isothermal cavitation is dictated by the cavitation number $\sigma$ (Equation (1) with constant vapor pressure $p_{v}$ ). In the context of cryogenic cavitation, the actual cavitation number needs to be defined as follows ${ }^{2}$ :

$$
\sigma=\frac{p_{\infty}-p_{v}\left(T_{c}\right)}{0.5 \rho_{l} U_{\infty}^{2}}
$$

where $p_{\infty}$ is the reference pressure, $U_{\infty}$ is the reference velocity, $\rho_{l}$ is liquid density, and $T_{c}$ is the temperature in the cavity. The local cavitation number can be related to the far-field cavitation number (based on the vapor pressure there) by the following first-order approximation ${ }^{2}$ :

$$
\frac{1}{2} \rho_{l} U_{\infty}^{2}\left(\sigma-\sigma_{\infty}\right)=\frac{d p_{v}}{d T}\left(T_{\infty}-T_{c}\right)
$$

Equation (2) clearly indicates that the cumulative effect of the aforesaid factors would tend to produce a noticeable rise in the local cavitation number and subsequently suppress the intensity of cavitation. Representative values of the vapor pressure gradients $\left(\mathrm{dp}_{\mathrm{v}} / \mathrm{dT}\right)$ in the operating temperature regime for liquid nitrogen and hydrogen are $20 \mathrm{kPa} / \mathrm{K}$ and $7 \mathrm{kPa} / \mathrm{K}$, respectively. For general background of cryogenic cavitation, we refer the reader to Brennen ${ }^{2}$.

Hord $^{23,24}$ conducted by far the most comprehensive experiments on cryogenic cavitation with liquid nitrogen and hydrogen, under different sets of inlet velocity and temperature conditions, and by employing a variety of geometries (hydrofoil and ogives of varying diameters). Temperature and pressure data in the cavitating region, which have been commonly employed for numerical validation ${ }^{25}$, was acquired over the geometries at regular spatial intervals by thermocouples and pressure sensors.

There have been limited computational studies for cryogenic cavitating flows. The key challenges for numerical computations are the presence of strong non-linearity in the energy equation and the temperature dependence of physical properties ${ }^{22}$ such as vapor pressure and density (as seen from Figure 1(B) and (C)). The main features of a few selected numerical studies are summarized in Table 1.

A transport-based cavitation model, proposed by Merkle et al. ${ }^{4}$, has been adopted in multiple efforts for non-cryogenic conditions. The same basic framework can also be used to simulate cryogenic cavitating flows, subject to proper modification of the model parameters to better reflect the transport properties of cryogenic fluids and physical mechanisms of the flow environment. Utturkar et al. ${ }^{15}$ showed that the accuracy of predictions is affected by the model parameters and we need to re-calibrate the model parameters to simulate cavitating flows in cryogenic conditions. As discussed earlier, the temperature dependent material properties also play a significant role in the predictions. These material properties are typically obtained from the models developed using the experimental data, and, naturally, contain uncertainties.

For computational verification and validation exercises, multiple aspects need to be addressed. One needs to ensure that the numerical representation of the analytical model reaches the correct solutions, at least asymptotically as the grids and time step sizes approach the limiting values; this is the so-called verification. One also needs to investigate whether and how a particular physical model can reproduce, or at least, satisfactorily approximate the observed phenomena and reproduce the experimental measurement; this is the so-called validation. The numerical approach employed in the present study has been previously tested, and documented, against a wide variety of problems ${ }^{15,19,20}$. Our focus here is to address the validation aspect, namely, to what extent a transport-based cavitation model can reproduce the physics, and how can we improve its performance. 
Specifically, we aimed at studying the impact of (i) the cryogenic cavitation model parameters and (ii) cryogenic fluid properties on the predictions, utilizing a global sensitivity analysis ${ }^{26}$. To facilitate the formulation of a suitable mathematical framework to probe the global sensitivity of the above-mentioned cavitation model and cryogenic fluid uncertainties, we first construct suitable surrogate models (Queipo et al. ${ }^{27}$ ). Since the fidelity of surrogate models is critical in determining the success of the sensitivity analysis and code validation exercise, we adopt different surrogate models to help ascertain the performance measures.

The practical utility of surrogate modeling for design, optimization and sensitivity analysis is well established ${ }^{27,28}$. There are many surrogate models (for example polynomial response surface, Kriging etc.) but the model that represents a particular function the best is not known a priori. Then, the predictions using different surrogate models have a certain amount of uncertainty. Goel et al. ${ }^{29}$ suggested that simultaneous use of multiple surrogate models may be beneficial to quantify and to reduce uncertainties in predictions. They proposed a weighted average surrogate model that was shown to represent a wide variety of test problems very well. In this study, we used four surrogate models, polynomial response surface approximation (PRS), Kriging (KRG), radial basis neural network (RBNN) and weighted average (WTA) surrogate model constructed using these three surrogates. These surrogate models are used to calibrate the model parameters of the present transport based cavitation model ${ }^{4}$ in cryogenic conditions.

Specifically, the objectives of this paper are:

i. To study the physical aspects of cavitation dynamics in cryogenic environment and perform code validation,

ii. To conduct a global sensitivity analysis to assess the sensitivity of the response to temperature dependent material properties and model parameters, and

iii. To calibrate the parameters of a transport-based cryogenic cavitation model for suitable flow conditions.

The organization of this paper is as follows. The governing equations and the numerical approach followed in this paper are described in Section II. The validation of the cavitation model is carried out in Section III. We present results of global sensitivity analysis to measure the relative importance of different model parameters and uncertainties in material properties and calibration of model parameters in Section IV. We recapitulate the major findings of the paper in Section V.

\section{Governing Equations and Numerical Approach}

The set of governing equations for cryogenic cavitation under the homogeneous-fluid modeling strategy comprises the conservative form of the Favre-averaged Navier-Stokes equations, the enthalpy equation, the $k-\varepsilon$ two-equation turbulence closure, and a transport equation for the liquid volume fraction. The mass-continuity, momentum, enthalpy, and cavitation model equations are given below:

$$
\begin{aligned}
& \frac{\partial \rho_{m}}{\partial t}+\frac{\partial\left(\rho_{m} u_{j}\right)}{\partial x_{j}}=0 \\
& \frac{\partial\left(\rho_{m} u_{i}\right)}{\partial t}+\frac{\partial\left(\rho_{m} u_{i} u_{j}\right)}{\partial x_{j}}=-\frac{\partial p}{\partial x_{i}}+\frac{\partial}{\partial x_{j}}\left[\left(\mu+\mu_{t}\right)\left(\frac{\partial u_{i}}{\partial x_{j}}+\frac{\partial u_{j}}{\partial x_{i}}-\frac{2}{3} \frac{\partial u_{k}}{\partial x_{k}} \delta_{i j}\right)\right] \\
& \frac{\partial}{\partial t}\left[\rho_{m}\left(h+f_{v} L\right)\right]+\frac{\partial}{\partial x_{j}}\left[\rho_{m} u_{j}\left(h+f_{v} L\right)\right]=\frac{\partial}{\partial x_{j}}\left[\left(\frac{\mu_{m}}{\operatorname{Pr}_{m}}+\frac{\mu_{t}}{\operatorname{Pr}_{t}}\right) \frac{\partial h}{\partial x_{j}}\right] \\
& \frac{\partial \alpha_{l}}{\partial t}+\frac{\partial\left(\alpha_{l} u_{j}\right)}{\partial x_{j}}=\dot{m}^{+}+\dot{m}^{-}
\end{aligned}
$$

where $\rho_{m}$ is the density of the fluid-vapor mixture, $u_{j}$ denotes the components of velocity, $p$ is pressure, $\mu_{m}, \mu_{t}$ are mixture laminar and turbulent viscosities, respectively, $h$ is sensible enthalpy, $f_{v}$ is the vapor mass fraction, $L$ is the latent heat of vaporization, $\operatorname{Pr}$ is the Prandtl number, $\alpha_{l}$ is the fraction of liquid in the mixture, and $\dot{m}^{+}, \dot{m}^{-}$are the source terms for the cavitation model. The subscript ' $t$ ' denotes turbulent properties, ' $l$ ' represents the liquid state, ' $v$ ' represents the vapor state, and ' $m$ ' denotes the 
mixture properties. The mixture property $\phi_{m}$, sensible enthalpy, and the vapor mass fraction are respectively expressed as

$$
\begin{aligned}
& \phi_{m}=\phi_{l} \alpha_{l}+\phi_{v}\left(1-\alpha_{l}\right) \\
& h=C_{P m} T \\
& f_{v}=\frac{\rho_{v}\left(1-\alpha_{l}\right)}{\rho_{m}}
\end{aligned}
$$

In the energy equation (5), we neglect the effects of kinetic energy and viscous dissipation terms $\left(O\left(\operatorname{Re}^{-0.5}\right), \operatorname{Re} \sim O\left(10^{6}\right)\right.$ ) because the temperature field in cryogenic cavitation is mainly dictated by the phenomenon of evaporative cooling.

\section{A. Transport-Based Cavitation Model}

Physically, the cavitation process is governed by thermodynamics and kinetics of the phase change process. The liquid-vapor conversion associated with the cavitation process is modeled through $\dot{m}^{+}$and $\dot{m}^{-}$terms in Equation (6), which represent condensation and evaporation, respectively. The particular form of these phase transformation rates, which in case of cryogenic fluids also dictate the heat transfer process, forms the basis of the cavitation model. The liquid-vapor condensation rates for the present transport based cavitation model $^{4}$ are:

$$
\dot{m}^{-}=\frac{C_{\text {dest }} \rho_{l} \operatorname{Min}\left(0, p-p_{v}\right) \alpha_{l}}{\rho_{v}\left(0.5 \rho_{l} U_{\infty}^{2}\right) t_{\infty}} \quad \dot{m}^{+}=\frac{C_{p r o d} \operatorname{Max}\left(0, p-p_{v}\right)\left(1-\alpha_{l}\right)}{\left(0.5 \rho_{l} U_{\infty}^{2}\right) t_{\infty}}
$$

where, $C_{\text {dest }}$ and $C_{\text {prod }}$ are the empirical model parameters controlling the evaporation and condensation rates, $p_{v}$ is the vapor pressure, $\rho_{v}, \rho_{l}$ are the vapor and liquid densities, $U_{\infty}$ is the reference velocity scale, and $t_{\infty}$ is the reference time scale, defined as the ratio of the characteristic length scale $D$ to the reference velocity scale $U_{\infty}\left(t_{\infty}=D / U_{\infty}\right)$. Merkle et al. ${ }^{4}$ validated this cavitation model with the experimental data for non-cryogenic fluids (e.g. water) and specified $C_{\text {dest }}=1.0$ and $C_{\text {prod }}=80.0$ as optimal model parameters (referred here as 'original' parameters). However, Utturkar ${ }^{20}$, and Hosangadi and Ahuja ${ }^{21}$ found that the previously calibrated values of the Merkle et al. ${ }^{4}$ cavitation model $\left(C_{\text {dest }}=1.0\right.$ and $C_{\text {prod }}=80.0$ ) are inadequate to provide a good match with the experimental data under the cryogenic condition. Consequently, Utturkar et al. ${ }^{15}$ suggested $C_{\text {dest }}=0.68$ and $C_{\text {prod }}=54.4$ via numerical experimentation, as more appropriate model parameters for liquid nitrogen. However, they noted difficulties in the simultaneous prediction of the temperature and pressure profiles on the surface of the test geometry. In this study, we refer these model parameters $\left(C_{d e s t}=0.68\right.$ and $\left.C_{\text {prod }}=54.4\right)$ as 'baseline' parameters.

\section{B. Thermodynamic Effects}

The evaporation and condensation processes result in absorption and release of the latent heat of vaporization that regulates the thermal effects. Furthermore, there is a significant variation in the physical properties $\left(\rho_{l}, \rho_{v}, p_{v}, \mu, C_{P}, K\right.$, and $\left.L\right)$ with temperature ${ }^{22}$ in the operating range that manifests coupling between different governing equations and underscores the importance of thermal effects in cryogenic cavitation. As indicated by phase diagram in Figure 1(D), the physical properties (liquid and vapor densities) are much stronger functions of temperature than pressure, and one can fairly assume the respective phase values on the liquid-vapor saturation curve at a given temperature.

To illustrate the impact of evaporation/condensation on the temperature in cavitation regime, we separate the latent heat terms in the energy equation (Equation (5)) onto the right-hand-side to obtain temperature-based form of the energy equation as follows. 


$$
\begin{aligned}
\frac{\partial}{\partial t}\left[\rho_{m} C_{P m} T\right]+\frac{\partial}{\partial x_{j}}\left[\rho_{m} u_{j} C_{P m} T\right] & =\frac{\partial}{\partial x_{j}}\left[C_{P m}\left(\frac{\mu_{m}}{\operatorname{Pr}_{L m}}+\frac{\mu_{t}}{\operatorname{Pr}_{t}}\right) \frac{\partial T}{\partial x_{j}}\right]- \\
& \underbrace{\left\{\frac{\partial}{\partial t}\left[\rho_{m}\left(f_{v} L\right)\right]+\frac{\partial}{\partial x_{j}}\left[\rho_{m} u_{j}\left(f_{v} L\right)\right]\right\}}_{\text {energy source/sink term }}
\end{aligned}
$$

As can be seen from Equation (11), the 'lumped' latent heat terms manifest as a non-linear source term into the energy equation and physically represent the latent heat transfer rate. The spatial variation of thermodynamic properties and the evaporative cooling effect are intrinsically embedded into this transportbased source term causing a coupling of all the governing equations.

\section{Speed of Sound (SoS) Model}

Numerical modeling of sound propagation is still an open question due to lack of dependable equation of state for liquid-vapor multiphase mixture. The speed of sound affects the numerical calculation via the pressure correction equation by conditionally endowing it with a convective-diffusive form in the mixture region. Past studies ${ }^{10,11,30,31}$ discuss in detail the modeling options, their impact and issues. The SoS model used here is outlined below.

$$
\mathrm{SoS}=C_{\rho}=C\left(1-\alpha_{l}\right)
$$

The density correction term in the continuity equation is thus coupled to the pressure correction term as shown below.

$$
\rho^{\prime}=C_{\rho} p^{\prime}
$$

In the pure liquid region, we recover the diffusive form of the pressure equation. Senocak and Shyy ${ }^{9,10}$ suggested an $O(1)$ value for the constant $C$ to expedite the convergence of the iterative computational algorithm. However, their recommendation is valid under normalized values for inlet velocity and liquid density. Since we employ dimensional form of equations for cryogenic fluids, we suggest an $\mathrm{O}\left(1 / U_{\infty}^{2}\right)$ value for $C$ (Utturkar ${ }^{20}$ ), which is consistent with the above suggestion in terms of the Mach number regime.

\section{Turbulence Model}

The $k-\varepsilon$ two-equation turbulence model with wall functions is presented as follows ${ }^{32}$ :

$$
\begin{aligned}
& \frac{\partial\left(\rho_{m} k\right)}{\partial t}+\frac{\partial\left(\rho_{m} u_{j} k\right)}{\partial x_{j}}=\mathrm{P}_{t}-\rho_{m} \varepsilon+\frac{\partial}{\partial x_{j}}\left[\left(\mu+\frac{\mu_{t}}{\sigma_{k}}\right) \frac{\partial k}{\partial x_{j}}\right] \\
& \frac{\partial\left(\rho_{m} \varepsilon\right)}{\partial t}+\frac{\partial\left(\rho_{m} u_{j} \varepsilon\right)}{\partial x_{j}}=C_{\varepsilon_{1}} \frac{\varepsilon}{k} \mathrm{P}_{t}-C_{\varepsilon_{2}} \rho_{m} \frac{\varepsilon^{2}}{k}+\frac{\partial}{\partial x_{j}}\left[\left(\mu+\frac{\mu_{t}}{\sigma_{\varepsilon}}\right) \frac{\partial \varepsilon}{\partial x_{j}}\right]
\end{aligned}
$$

The turbulence production $\left(\mathrm{P}_{t}\right)$ and the Reynolds stress tensor are defined as:

$$
\begin{aligned}
& \mathrm{P}_{t}=\tau_{i j} \frac{\partial u_{i}}{\partial x_{j}} ; \tau_{i j}=-\overline{\rho_{m} u_{i}^{\prime} u_{j}^{\prime}} \\
& \overline{\rho_{m} u_{i}^{\prime} u_{j}^{\prime}}=\frac{2 \rho_{m} k \delta_{i j}}{3}-\mu_{t}\left(\frac{\partial u_{i}}{\partial x_{j}}+\frac{\partial u_{j}}{\partial x_{i}}\right)
\end{aligned}
$$

The parameters for this model, namely, $C_{\varepsilon 1}=1.44, C_{\varepsilon 2}=1.92, \sigma_{\varepsilon}=1.3, \sigma_{k}=1.0$ are adopted from the equilibrium shear flow calibration ${ }^{33}$. The turbulent viscosity is defined as: 


$$
\mu_{t}=\frac{\rho_{m} C_{\mu} k^{2}}{\varepsilon}, C_{\mu}=0.09
$$

Of course, the turbulence closure and the eddy viscosity levels can affect the outcome of the simulated cavitation dynamics especially in case of unsteady simulations. For detailed investigations in this regard, for cavitating flow computations, we refer to recent works by Wu et al. ${ }^{34}$ and Utturkar et al. ${ }^{19}$.

\section{E. Numerical Approach}

The governing equations are numerically solved using a CFD code STREAM ${ }^{35}$ based on a pressurebased algorithm and the finite-volume approach. We use multi-block, structured, curvilinear grids to solve the problems in this paper. The viscous terms are discretized by second-order accurate central differencing while the convective terms are approximated by the second-order accurate controlled variations scheme $(\mathrm{CVS})^{36}$. The use of CVS scheme prevents oscillations under sharp gradients caused by the evaporation source term in the cavitation model, while retaining second order of formal accuracy. The pressure-velocity coupling is implemented through the extension of the SIMPLEC ${ }^{37}$ type of algorithm cast in a combined Cartesian-contravariant formulation ${ }^{35}$ for the dependent and flux variables respectively, followed by adequate relaxation for each governing equation, to obtain steady-state results. The temperature dependent material properties are updated from the NIST $^{22}$ database at the end of each computational iteration.

\section{Basic Cryogenic Cavitation Model Performance}

In this section, we describe the test geometry and numerical setup used to validate the present transport based cryogenic cavitation model ${ }^{4}$. Specifically, we study the role of model parameters, boundary conditions and thermal effects.

\section{A. Test Geometry and Boundary Conditions}

We simulate flow over a 2-D hydrofoil in cryogenic environment which serves as a benchmark problem for validating the cryogenic cavitation models. $\operatorname{Hord}^{23}$ experimentally investigated the flow over this geometry inside suitably designed wind-tunnels. He reported average pressure and temperature data at five probe locations over the body surface for different cases that are referenced alpha-numerically. We employ Case '290C' ( $\mathrm{Re}=9.1 \times 10^{6}, \sigma_{\infty}=1.7, T_{\infty}=83.06 \mathrm{~K}$, liquid nitrogen fluid) that is centrally located in the temperature range, for model validation.

A simplified computational domain and the boundary conditions for this problem are shown in Figure 2. The computational grid consists of 320x70 non-uniformly distributed grid points such that the cavitation regime is adequately resolved and the deployment of wall functions near the no-slip boundary conditions is allowed (Utturkar et al. ${ }^{15}$ ). The inlet boundary conditions are implemented by stipulating the values of the velocity components, phase fraction, temperature, and turbulence quantities from the experimental data ${ }^{23}$. At the walls, pressure, phase fraction, and turbulence quantities are extrapolated, along with applying the no-slip (in the form of the wall function ${ }^{37}$ ) and adiabatic conditions on the velocity and temperature, respectively. Pressure and other variables are extrapolated at the outlet boundaries while enforcing global mass conservation by rectifying the outlet velocity components. In addition, we hold the pressure at the reference point (illustrated in the experimental report ${ }^{23}$ ) constant at the reference value specified by the experiments.

The quality of the predictions is measured by comparing computed and experimentally known pressure and temperature values at each of the five probe locations on the surface of hydrofoils. Corresponding $L_{2}$ norms of the differences between computed and experimental values of pressure $\left(P_{\text {diff }}\right)$ and temperature $\left(T_{\text {diff }}\right)$ are used as metrics that are desired to be low to obtain good prediction quality.

\section{B. Pressure and Temperature Predictions}

Firstly, we discuss the results of predictions using present transport based cavitation model ${ }^{4}$ with the baseline parameters and highlight the influence of thermal effects. We compare the predicted temperature and pressure profiles on the surface of the hydrofoil with the experimental data in Figure 3 . The difference of pressure on the surface of the hydrofoil and free stream vapor pressure $\left(p-p_{v}\left(T_{\infty}\right)\right)$, and the difference of pressure on the surface of the hydrofoil and the actual vapor pressure (based on temperature, $p-p_{v}(T)$ ) for baseline case are shown in Figure 3(A). The cavitation in cryogenic environment differs from non- 
cryogenic environment in two ways: (i) under-shoot at the leading edge of the hydrofoil indicates slower pressure recovery in cryogenic environment than that observed in the non-cryogenic environment, and (ii) the vapor pressure in the cavity in cryogenic environment is not constant (continuous increase) due to the variation in temperature. This increase in vapor pressure (as marked by $\Delta p_{v}$ in Figure 3(A)) is attributed to the variation in temperature (Figure 1). The change in vapor pressure affects the cavitation source terms and resultant liquid-vapor fraction which impacts the source terms in energy equation to enforce coupling of thermal effects in governing equations. To contrast the thermal effect on the cavitation dynamics, we also show a solution obtained by assigning a zero latent heat in Figure 3(A). With zero latent heat and with an adiabatic wall condition the fluid field exhibits a constant temperature throughout, resulting in a constant vapor pressure. This isothermal cavitation case yields a substantially larger cavity, which is quite different from the experimental measurement. Figure 3(A) highlights the importance of the thermal effect on cryogenic cavitation. It also depicts the range of variations in vapor pressure reflecting the temperature distributions inside the cavity.

The temperature on the surface of hydrofoil in cavitating conditions is shown in Figure 3(B). The significant drop in temperature near the leading edge of the cavity is explained as follows. The phase change, as modeled, is dictated by the vapor pressure. When the local pressure in the flow falls below the vapor pressure, evaporation begins instantaneously as indicated by the transport model. This results in absorption of the latent heat of vaporization to facilitate the phase change. However, unlike boiling heat transfer, where heat is continuously supplied through an external heat source, the heat transfer in cavitating flow largely stems from the convective and conductive heat transfer, and the latent heat release/absorption within the fluid, with external heat source playing minor roles. Consequently, a decrease in fluid temperature is observed in the cavity region. As we approach the cavity closure region, the condensation of fluid releases latent heat increasing the fluid temperature locally. Furthermore, since the condensation process is dictated by the vapor pressure (with the local temperature effect exerted indirectly via the change in vapor pressure in response to the temperature field), the rate of latent heat release can be fast in comparison to the rate of convective and conductive heat transfer and consequently in simulations, we observe an "overshoot" in temperature profile. The experiments also show an increase in temperature of the fluid in the closure region but probably due to the lack of sufficient number of probes on the surface, the existence of the overshoot could not be ascertained.

Overall, the pressure predictions on the hydrofoil surface follow the same trends as observed in experiments. However, we note differences in predictions with experimental data near the closure region of the cavity.

\section{Role of Cavitation Model Parameters}

Next, we compare the performance of present transport based cavitation model ${ }^{4}$ with the original and the baseline parameters to illustrate the impact of model parameters on the predictions. The pressure and temperature profiles on the surface of the hydrofoil along with the experimental data are shown in Figure 4. The predicted pressure profiles using the baseline parameters are in much better agreement with the experimental data compared to those obtained with the original parameters while the temperature profile using the two sets of parameters are comparable. The numerical quantification of the $L_{2}$ norm of the differences in pressure $\left(P_{\text {diff }}\right)$ and temperature predictions $\left(T_{\text {diff }}\right)$ given in Table 2, clearly shows significant improvements in the prediction and highlights the role of model parameters on the predictions. The present cavitation model, using the baseline parameters (Utturkar et al. ${ }^{15}$ ), predicts a smaller cavity compared to that obtained with the original parameters (Merkle et al. ${ }^{4}$ ). The predictions have significant variations with the model parameters. While the baseline model parameters improve predictions significantly compared to the original model parameters, we note significant differences in the temperature and pressure predictions, particularly in the cavity closure region.

\section{Impact of Boundary Conditions}

To investigate the discrepancy between experimental and predicted surface pressure and temperature profiles, we evaluate the impact of different thermal boundary conditions on the predictions. While all the walls on the wind tunnel are modeled as adiabatic, the hydrofoil surface is modeled as either adiabatic (Neumann boundary) or constant temperature (Dirichlet boundary) wall. The temperature profile required for implementing Dirichlet boundary condition is obtained by inter-/extra-polating the experimental temperature at five probe locations on the surface of hydrofoil. 
The pressure and temperature profiles on the surface of hydrofoil from the simulations with different thermal boundary conditions are compared with the experimental data $\left(\operatorname{Hord}^{23}\right)$ in Figure 5. The introduction of heat transfer through the hydrofoil surface by Dirichlet boundary condition has little influence on the pressure distribution. With the given Reynolds number, the heat transfer at the hydrofoil surface is relatively small compared to the impact of latent heat, and subsequently only minor variations in the vapor pressures are observed. In the cavity closure region, the latent heat released during condensation cannot be redistributed via convection and conduction fast enough, resulting in an overshoot in temperature there. The temperature profile on the first computation point above the hydrofoil surface, shown in Figure $5(\mathrm{C})$, also indicates that the effect of heat transfer due to Dirichlet boundary condition is largely restricted to the boundary and has minimal influence on the flow inside the cavity.

Overall, it can be said that the effect of thermal boundary condition on the hydrofoil surface has little impact on the performance of the present cryogenic cavitation model.

\section{Surrogates-based Global Sensitivity Assessment and Calibration}

Since minor changes in flow environment can lead to substantial changes in the predictions in cryogenic environment (Utturkar et al. ${ }^{15}$ ), it is imperative to appraise the role of model parameters and uncertainties in material properties on the predictions. In this section, we characterize the parameters which significantly affect predictions using surrogate-based global sensitivity analysis (GSA) and then calibrate the cryogenic cavitation model parameters. Goel et al. ${ }^{29}$ have shown that multiple surrogate models are beneficial to avoid large errors due to uncertainty in surrogates and to ascertain uncertainty in predictions in design space. So we use polynomial response surface approximation, Kriging, radial basis neural network, and a weighted average surrogate model for approximation of response.

\section{A. Global Sensitivity Analysis}

We employ variance-based, non-parametric GSA method, proposed by Sobol ${ }^{26}$ (refer to Appendix 1), to evaluate the sensitivity of predictions with respect to model parameters and material properties. In this method, the response function is decomposed into additive functions of variables and interactions among variables. This allows the total variance $(V)$ in the response function to be expressed as a combination of the main effect of each variable $\left(V_{i}\right)$ and its interactions with other variables $\left(V_{i j}\right)$. The sensitivity of the response function with respect to any variable is measured by computing its sensitivity indices. The sensitivity indices of main effect $\left(S_{i}\right)$ and total effect ( $S_{i}^{\text {total }}$ ) of a variable are given as follows:

$$
S_{i}=V_{i} / V, \quad S_{i}^{\text {total }}=\left(V_{i}+V_{i Z}\right) / V
$$

We can study the influence of uncertainty in different material properties $\rho_{v}, \rho_{l}, K, C_{p}$, and $L$, and model parameters $C_{\text {prod }}, C_{d e s t}$, and $t_{\infty}$. However, to keep Re (Reynolds number based on upstream flow) and $\sigma_{\infty}$ (cavitation number based on upstream flow) constant for the given case and account for most influential material properties, we choose $C_{\text {dest }}, t_{\infty}, \rho_{v}$, and $L$ as variables. The predictions of the cryogenic cavitation models are represented by root mean square (RMS) values of the hydrofoil surface temperature $\left(T_{R M S}\right)$ and pressure coefficient $\left(C_{p R M S}\right)$, where $C_{p}$ is defined as,

$$
C_{p}=\left(p-p_{\infty}\right) /\left(0.5 \rho_{l} U_{\infty}^{2}\right)
$$

The model parameters, $C_{d e s t}$ and $t_{\infty}$, are perturbed on either side of their baseline values ( $C_{\text {dest }}=0.68 ; t_{\infty}=1.82 \times 10^{-3}$ ) by $15 \%$ and the material properties $\rho_{v}$ and $L$ are perturbed within $10 \%$ of the value they assume from the NIST database ${ }^{22}$. The ranges of the variables are given in the Table 3 .

To curb the computational cost of performing GSA, we develop surrogate models of the objectives. 49 data points using face-centered cubic composite design (FCCD, 25 points) and orthogonal arrays (OA, 24 points) are sampled to construct surrogate models and four points are used to validate the surrogate models.

We evaluate $T_{R M S}$ and $C_{p_{R M S}}$ for each data point using CFD simulations and construct polynomial response surface (PRS) and Kriging models (refer to Appendix 2) of both responses in normalized variable space. 
All variables are normalized such that ' 0 ' corresponds to the minimum value and ' 1 ' corresponds to the maximum value of the variable. Normalized variable are denoted by a superscript '*'. We use $2^{\text {nd }}$ order polynomials for PRS and a linear polynomial trend model with Gaussian correlation function for Kriging approximation. Relevant details of the two surrogate models are summarized in Table 4 and predictions on the four test points are given in Table 5. Low errors in approximations on training and test points indicate that both PRS and Kriging approximate the objectives very well.

The Kriging parameters associated with Gaussian correlation function for the two responses are given in Table 6 and the linear trend models are given as follows:

$$
\begin{aligned}
& C_{p R M S}=0.108+0.577 C_{d e s t}^{*}-0.450 \rho_{v}^{* *}-0.039 L^{* *}-0.618 t_{\infty}^{*} \\
& T_{R M S}=-0.036-0.593 C_{d e s t}^{*}+0.307 \rho_{v}^{* *}-0.110 L^{* *}+0.616 t_{\infty}^{*}
\end{aligned}
$$

The PRS approximations of the responses are given by Equations (22) and (23).

$$
\begin{aligned}
C_{p R M S}= & 1.675+0.077 C_{\text {dest }}^{*}-0.061 \rho_{v}^{* *}-0.082 t_{\infty}^{*}-0.007 C_{\text {dest }}^{*} \rho_{v}^{* *} \\
& -0.012 C_{\text {dest }}^{*} t_{\infty}^{*}+0.011 \rho_{v}^{* * 2}+0.009 \rho_{v}^{* *} t_{\infty}^{*}-0.004 L^{* * 2}+0.013 t_{\infty}^{* 2} \\
T_{R M S}= & 82.537-0.243 C_{d e s t}^{*}+0.107 \rho_{v}^{* *}-0.048 L^{* *}+0.220 t_{\infty}^{* 2}+0.024 C_{d e s t}^{* 2} \\
& -0.016 C_{\text {dest }}^{*} \rho_{v}^{* *}-0.015 C_{d e s t}^{* 2} L^{* *}+0.013 \rho_{v}^{* *} L^{* *}+0.018 \rho_{v}^{* *} t_{\infty}^{*}+0.019 L^{* *} t_{\infty}^{*}
\end{aligned}
$$

While relatively low importance of latent heat $(L)$ can be deduced upfront by inspecting the coefficients in the PRS and the Kriging parameters, a formal global sensitivity analysis is conducted to quantify the contribution of each design variable on the variability of our responses $\left(C_{p_{R M S}}\right.$ and $T_{R M S}$ ). The sensitivity indices of main and interaction effects of different variables on $C_{p_{R M S}}$ and $T_{R M S}$ are shown in Figure 6 and Figure 7, respectively. It can be seen from the pie-charts that the impact of model parameters on the variability of $C_{p_{R M S}}$ and $T_{R M S}$ is equally important and more than the variability due to material properties. While the effect of latent heat $(L)$ on the variability of the RMS surface pressure coefficient and temperature within the selected uncertainty range is negligible, though the overall importance of latent heat in the coupling of thermal effects was illustrated in Figure 3. The effect of variability in vapor density $\rho_{v}$ is significant, which means that the performance of cryogenic cavitation model is susceptible to the inaccuracies in the prediction of the temperature dependent vapor density $\rho_{v}$ and we should pay more attention to develop accurate models of $\rho_{v}$. The interaction of different variables did not influence the variability in the predictions. The results from both surrogate models are in good agreement which improves our confidence in results. For this case, the results clearly are not dependent on the particular form of surrogate models.

The results of sensitivity estimates indicate that the variables, unlike $L$, which appear either in $\dot{m}^{-}$or $\dot{m}^{+}$may tend to register greater influence on the computed results. Thus, intuitively, reference velocity $U_{\infty}$ and liquid density $\rho_{l}$, which are omitted from the present GSA, are expected to induce large variability in the computation, as compared to other omitted properties such as thermal conductivity $K$ and specific heat $C_{p}$. Furthermore, as depicted by Figure 6 and Figure 7, the impact of different parameters is expected to be same on pressure and temperature due to the tight coupling between various flow variables.

\section{B. Calibration of Cryogenic Cavitation Model}

Next, we calibrate the present cryogenic cavitation model for cryogenic environments using the benchmark hydrofoil case '290C'. We assume the temperature dependent material properties obtained from the NIST database ${ }^{22}$ to be accurate and treat model parameters $C_{d e s t}$ and $t_{\infty}$ as parameters to be tuned. The performance of the cavitation model is determined by computing the $L_{2}$ norm of the difference in the experimental data and predicted data at five probe points for pressure $\left(P_{\text {diff }}\right)$ and temperature $\left(T_{\text {diff }}\right)$. The objective of the calibration is to minimize the deviation from the experimental data $P_{\text {diff }}$ and $T_{\text {diff. }}$. As shown 
in Figure 8, the parameters which yield good pressure predictions (low $P_{\text {diff }}$ ) yield large errors in temperature predictions (high $T_{\text {diff }}$ ) and vice-versa (low $T_{\text {diff }}$ but high $P_{\text {diff }}$ ). So this problem of calibrating parameters is cast as a multi-objective optimization to simultaneously minimize $P_{\text {diff }}$ and $T_{\text {diff }}$.

\section{(i) Construction of Multiple Surrogate Models}

We construct multiple surrogates using 55 data points selected via FCCD (9 points) and Latin hypercube sampling (46 points) in the design space (refer to Table 3 for range of variables). For each data point, we evaluate objectives $P_{\text {diff }}$ and $T_{\text {diff }}$ using CFD simulations and the range of objectives is given in Table 7. We construct PRS, Kriging (KRG), RBNN, and WTA surrogate models (refer to Appendix 2 for a brief description of different surrogate models). Here, we use quintic polynomials for PRS, a linear trend model and Gaussian correlation function for Kriging. RBNN input parameter 'goal' is taken as 2e-2 and 3e5 , and 'spread' is taken as 0.55 and 0.45 for $P_{\text {diff }}$ and $T_{\text {diff, }}$ respectively. The weights associated with different surrogates in WTA model are given in Table 8. Different measures of the quality of surrogate models are summarized in Table 9. We observe that all surrogate models represent the two objective functions adequately. Relatively, Kriging is the best surrogate model as this has the lowest PRESS and RBNN (Appendix 3) is the worst of all the surrogates. The weights of different surrogates in WTA model also reflect the same. The WTA model obtained by averaging different surrogate models performs comparably with the best surrogate.

We show the contours of $P_{\text {diff }}$ and $T_{\text {diff }}$ in entire design space using different surrogate models in Figure 9 and Figure 10, respectively. All surrogate models have largely similar performance in entire design space though there are subtle differences in some regions. We see that the region that corresponds to low $P_{\text {diff }}$ corresponds to high $T_{\text {diff }}$ and vice-versa. This clearly establishes the need of performing a multi-objective optimization to simultaneously minimize both $P_{\text {diff }}$ and $T_{\text {diff. }}$.

\section{(ii) Preliminary Calibration and Design Space Refinement}

We simultaneously minimize $P_{d i f f}$ and $T_{\text {diff }}$ to calibrate the present cavitation model. This problem is reduced to a single objective optimization problem by combining the two difference metrics using different weights (weighted sum strategy ${ }^{38}$ ) or by treating one difference metric as objective function and the second difference metric as constraint function $\left(\varepsilon\right.$-constraint strategy $\left.{ }^{39}\right)$. We obtain many candidate Pareto optimal solutions by varying the weights for weighted sum strategy and constraint values for $\varepsilon$-constraint strategy. After removing dominated and duplicate solutions from the set of candidate solutions, the function space and variable space illustration of Pareto optimal front (POF) obtained through different surrogate models is shown in Figure 11. We observe that different POF obtained by using multiple surrogate models are close to one another in the function space but have significant disparity in the variable space. Nevertheless, all surrogate models predict that a small increase in $T_{\text {diff }}$ will lead to significant reduction in the $P_{\text {diff }}$ (Figure 11(A)). Based on the information from multiple surrogate models, we identify the encircled region in POF as the region of interest (compromise zone) where we expect the predictions to have low $P_{\text {diff }}$ and low $T_{\text {diff }}$. The variable space representation of the compromise zone (POF data on boundary for PRS, and interior points for KRG and RBNN) is shown in Figure 11(B). The ranges of the variables in the compromise zone are given in Table 10.

Once we have identified the region of interest, we can improve the accuracy of surrogate models by sampling additional points in the region and reconstructing surrogate models only in the region of interest (Mack et al. ${ }^{40}$ ). To facilitate the same, we sample 22 additional points at the Pareto optimal solutions obtained from different surrogate models in compromise zone. The location of sampled points is shown in Figure 12. The ranges of the objectives $P_{\text {diff }}$ and $T_{\text {diff }}$ based on 30 points ( 8 pre-existing points and 22 additional sampled points) are given in Table 11.

We construct surrogate models using 30 points in the region of interest. As before we use a linear trend model and Gaussian correlation function for Kriging but we employ quartic polynomials for PRS and RBNN input parameter 'spread' is fixed at 0.5 and 'goal' is taken as $3 \mathrm{e}-2$ and $6.35 \mathrm{e}-4$ for $P_{\text {diff }}$ and $T_{\text {diff, }}$, respectively. The weights associated with different surrogates in WTA model are given in Table 12 . Different measures of the quality of surrogate models, summarized in Table 13, suggest that all surrogate models approximated the actual response accurately.

Had we followed the conventional approach of selecting the best surrogate model for one data set and using the same surrogate model for all other sets, we would have used Kriging as the best surrogate model. However, as can be seen from the results, the Kriging model is no the best surrogate model for refined 
design space. Also we would not have sampled the points near the boundary for refined design space. These results demonstrate the advantages reaped from the simultaneous use of multiple surrogates.

\section{(iii) Final Calibration of Cryogenic Cavitation Model}

The Pareto optimal solutions in the refined design space obtained via weighted sum strategy and $\varepsilon$ constraint strategy are shown in Figure 13 along with the POF obtained in entire design space. The range of the POF is truncated to highlight the region of interest. While the predictions of Kriging and RBNN surrogates fitted in complete design space tend to under predict the objectives, PRS over predict the responses (compare solid lines to the diamonds). Agreement between POF from different surrogates fitted to data from the compromise zone is better (dotted lines). WTA model predicts the POF quite accurately for both datasets.

We select two trade-off designs on the POF obtained from the data in the new compromise zone for validation. The designs are selected such that high reduction in $P_{\text {diff }}$ can be realized without incurring significant deterioration of $T_{\text {diff. }}$. The variables, predicted and actual $P_{\text {diff }}$ and $T_{\text {diff }}$ values for different surrogate models at trade-off designs are given in Table 14. The errors in predictions of $P_{\text {diff }}$ and $T_{\text {diff }}$ are reasonably small, though we observed large variability in Kriging predictions. The improvements in the predictions of cavitation model through this surrogate modeling calibration are quantified in Table 15 . We obtain a $65 \%$ reduction in $P_{\text {diff }}$ by allowing $3.5 \%$ increase in $T_{\text {diff. }}$ The improvements after second refinement of variable space are small and hence no further refinement in design space is sought. The surface pressure and temperature profiles obtained using the calibrated values are compared to the results obtained with the baseline and original parameters of present transport based cavitation model ${ }^{4}$ in Figure 14. The improvements in the surface pressure predictions are obvious whereas the deterioration in the temperature predictions is small.

From cavitation dynamics point of view, the main issue with the predictions using baseline parameters was the poor prediction of the cavity closure region. The optimal model parameters reduce the evaporation source term and increase the condensation source term. This change brings favorable changes in the cavity closure region by increasing the condensation rate and hence faster recovery of the pressure as was observed in experiments.

\section{Validation of the Calibrated Cavitation Model}

The calibrated model parameters of the present cryogenic cavitation model are validated by simulating additional benchmark cases for liquid nitrogen and liquid hydrogen. The cases considered in the present paper are enlisted in Table 16. For both liquid nitrogen and hydrogen, the optimal parameters were taken as $C_{\text {dest }}=0.85 \times\left. C_{\text {dest }}\right|_{\text {baseline }}$ and $t_{\infty}=0.89 \times\left. t_{\infty}\right|_{\text {baseline }}$, where baseline model parameters are the same as suggested by Utturkar et al. ${ }^{15}$. The optimal model parameters for different fluids are given in Table 17. The surface pressure and temperature profiles for different cases along with the experimental data for liquid N2 cases and liquid $\mathrm{H} 2$ case are shown in Figure 15 and Figure 16, respectively. We also show the predictions using the baseline (Utturkar et al. ${ }^{15}$ ) and original model parameters (Merkle et al. ${ }^{4}$ ). The model with recalibrated model parameters captures the experimental data significantly better than the model with baseline or original parameters. For liquid hydrogen, though the surface pressure predictions improved significantly compared to the baseline parameters, there is scope for further improvement by seeking optimal set of parameters.

Nevertheless, the results confirm the improvements in the predictions by using optimized model parameters.

\section{Conclusions}

In this paper, we presented results of code validation and model improvement of a transport-based cryogenic cavitation model using benchmark experimental data for 2-D hydrofoils provided by $\mathrm{Hord}^{23}$. We used surrogate-based global sensitivity analysis to study the role of model parameters and uncertainties in temperature dependent material properties. The model parameters originally used in present transport based cavitation model $^{4}$ were calibrated for cryogenic environment using multiple surrogates, optimization and design space refinement techniques. The main conclusions of the paper are as follows.

i. Performance of the present approach was more affected by cavitation model parameters than uncertainty in material properties. However, the high sensitivity index associated with temperature dependent vapor density indicated that the latter might have significant impact on predictions too. 
ii. Simultaneous use of multiple surrogate models evidently helped in increasing confidence in the results of global sensitivity analysis. We also observed that design space refinement based on multiple surrogate models helped bound the optimal model parameters. Furthermore, predictions using weighted average surrogate models were more accurate than individual surrogate models.

iii. The impact of thermal boundary conditions on the prediction of flow was apparently not significant. However, the thermal effect caused by the phase change clearly affects the cavitation dynamics including the vapor pressure and, consequently, the cavity size.

iv. Optimal model parameters for present transport based cavitation model ${ }^{4}$ were $C_{\text {dest }}=0.85 \times\left. C_{\text {dest }}\right|_{\text {baseline }}$ and $t_{\infty}=0.89 \times\left. t_{\infty}\right|_{\text {baseline }}$. Correspondingly, for liquid nitrogen, optimal values were $C_{\text {dest }}=0.578$ and $t_{\infty}=2.36 \times 10^{-3}$ and for liquid hydrogen, values were $C_{\text {dest }}=0.697$ and $t_{\infty}=9.68 \times 10^{-4}$. The choice of these parameters increased the importance of condensation source term which improved predictions in cavity closure region.

In view of the persistent discrepancies between measurements and computations, further modeling development at a conceptual level should be pursued to improve the physical representation of the present cavitation model, and more experimental investigation is needed to better quantify the measurement uncertainty and offer insight into flow structures.

\section{Acknowledgements}

The present efforts have been supported by the Institute for Future Space Transport, under the NASA Constellation University Institute Program (CUIP), Ms. Claudia Meyer program monitor. This material is based upon work supported by the National Science Foundation under Grant No. 0423280. We acknowledge the valuable collaboration with Dr. Yogen Utturkar and Dr. Jiongynag Wu during their doctoral research.

\section{References}

1. Batchelor, GK, An Introduction to Fluid Dynamics, Cambridge University Press (1967).

2. Brennen, CE, Hydrodynamics of Pumps, Oxford University Press (1994).

3. NASA online facts, http://www-pao.ksc.nasa.gov/kscpao/nasafact/count2.htm (1991).

4. Merkle, CL, Feng, J, and Buelow, PEO, "Computational Modeling of Dynamics of Sheet Cavitation," Proceedings of $3^{\text {rd }}$ International Symposium on Cavitation, Grenoble, France (1998).

5. Ahuja, V, Hosangadi, A, and Arunajatesan, S, "Simulations of Cavitating Flows Using Hybrid Unstructured Meshes," Journal of Fluids Engineering 123, 331 (2001).

6. Venkateswaran, S, Lindau, JW, Kunz, RF, and Merkle, CL, "Computation of Multiphase Mixture Flows with Compressiblity Effects," Journal of Computational Physics 180, 54 (2002).

7. Kunz, RF, Boger, DA, Stinebring, DR, Chyczewski, TS, Lindau, JW, and Gibeling, HJ, "A Preconditioned Navier-Stokes Method for Two-phase Flows with Application to Cavitation," Computers and Fluids 29, 849 (2000).

8. Athavale, MM, and Singhal, AK, "Numerical Analysis of Cavitating Flows in Rocket Turbopump Elements," AIAA Paper 2001-3400 (2001)

9. Senocak, I, and Shyy, W, "A Pressure-based Method for Turbulent Cavitating Flow Computations," Journal of Computational Physics, 176, 363 (2002).

${ }^{10 .}$ Senocak, I, and Shyy, W, "Interfacial Dynamics-Based Modeling of Turbulent Cavitating Flows, Part1: Model Development and Steady-State Computations," International Journal for Numerical Methods in Fluids 44, 975 (2004).

11. Senocak, I, and Shyy, W, "Interfacial Dynamics-Based Modeling of Turbulent Cavitating Flows, Part2: Time-Dependant Computations," International Journal for Numerical Methods in Fluids 44, 997 (2004).

12. Kubota, A, Kato, H, and Yamaguchi, H, "A New Modeling of Cavitating Flows: A Numerical Study of Unsteady Cavitation on a Hydrofoil Section," Journal of Fluid Mechanics, 240, 59 (1992).

13. Edwards, JR, Franklin, RK, and Liou, MS, "Low-Diffusion Flux Splitting Methods for Real Fluid Flows with Phase Transitions," AIAA Journal 38(9), 1624 (2000). 
14. Ventikos, Y, and Tzabiras, G, "A Numerical Method for Simulation of Steady and Unsteady Cavitating Flows," Computers and Fluids 29, 63 (2000).

${ }^{15 .}$ Utturkar, Y, Goel, T, Thakur, SS, and Shyy, W, "Computational Modeling of Thermodynamic Effects in Cryogenic Cavitation," (submitted to International Journal of Multi-Phase Flow).

16. Wang, G, Senocak, I, Shyy, W, Ikohagi, T, and Cao, S, "Dynamics of Attached Turbulent Cavitating Flows," Progress in Aerospace Sciences 37, 551 (2001).

17. Senocak, I, and Shyy, W, "Evaluation of Cavitation Models for Navier-Stokes Computations," ASME Paper FEDSM2002-31011 (2002).

18. Preston, A, Colonius, T, and Brennen, CE, "Towards Efficient Computation of Heat and Mass Transfer Effects in the Continuum Model for Bubbly Cavitating Flows," Proceedings of $4^{\text {th }}$ International Symposium on Cavitation, Pasadena, California (2001).

${ }^{19 .}$ Utturkar, Y, Wu, J, Wang, G, and Shyy W, "Recent Progress in Modeling of Cryogenic Cavitation for Liquid Rocket Propulsion," (submitted to Progress in Aerospace Sciences).

${ }^{20 .}$ Utturkar, Y, "Computational Modeling of Thermodynamic Effects in Cryogenic Cavitation", Ph.D. Dissertation, University of Florida, Gainesville FL, (2005).

${ }^{21 .}$ Hosangadi, A, and Ahuja, V, "Numerical Study of Cavitation in Cryogenic Fluids", Journal of Fluids Engineering, 127:267-281, (2005).

22. Lemmon, EW, McLinden, MO, and Huber, ML, REFPROP: Reference Fluid Thermodynamic and Transport Properties, NIST Standard Database 23, version 7.0 (2002).

${ }^{23 .}$ Hord, J, "Cavitation in Liquid Cryogens, II-Hydrofoil," NASA CR-2156 (1973).

${ }^{24 .}$ Hord, J, "Cavitation in Liquid Cryogens, III - Ogives," NASA CR-2242 (1973).

${ }^{25 .}$ Hosangadi, A, and Ahuja, V, "A Generalized Multi-phase Framework for Modeling Cavitation in Cryogenic Fluids," AIAA Paper 2003-4000 (2003).

26. Sobol, IM, "Sensitivity Analysis for Nonlinear Mathematical Models," Mathematical Modeling \& Computational Experiment 1(4), 407 (1993).

27. Queipo, NV, Haftka, RT, Shyy, W, Goel, T, Vaidyanathan, R, and Tucker, PK, "Surrogate Based Analysis and Optimization”, Progress in Aerospace Sciences, 41:1-28 (2005).

${ }^{28 .} \mathrm{Li}$, W, and Padula, S, "Approximation Methods for Conceptual Design of Complex Systems", Proceedings of $11^{\text {th }}$ International Conference on Approximation Theory, (eds. Chui C, Neaumtu M, Schumaker L), (2004).

29. Goel, T, Haftka, RT, Shyy, W, and Queipo, NV, "Ensemble of Multiple Surrogates", accepted for publication in Structural and Multidisciplinary Optimization, (2006).

${ }^{30 .}$ Senocak, I, and Shyy, W, "Computations of Unsteady Cavitation with a Pressure-based Method," ASME Paper FEDSM2003-45009 (2003).

${ }^{31 .}$ Wu, J, Utturkar, Y, Senocak, I, Shyy, W, and Arakere, N, "Impact of Turbulence and Compressibility Modeling on Three-Dimensional Cavitating Flow Computations," AIAA Paper 2003-4264 (2003).

32. Launder, BE, and Spalding, DB, "The Numerical Computation of Turbulent Flows," Computer Methods in Applied Mechanics and Engineering 3, 269 (1974).

33. Shyy, W, Thakur, SS, Ouyang, H, Liu, J, and Blosch, E, "Computational Techniques for Complex Transport Phenomenon", Cambridge University Press, United Kingdom, (1997).

${ }^{34 .} \mathrm{Wu}$, J, Wang, G, and Shyy, W, "Time-dependent Turbulent Cavitating Flow Computations with Interfacial Transport and Filter-based Models", International Journal of Numerical Methods in Fluids, 49(7):739-761, (2005).

35. Thakur, SS, Wright, J, and Shyy, W, "STREAM: A Computational Fluid Dynamics and Heat Transfer Navier-Stokes Solver. Theory and Applications," Streamline Numerics, Inc. and Computational Thermo-Fluids Laboratory, Department of Mechanical and Aerospace Engineering Technical Report, Gainesville, Florida (2002).

36. Shyy, W, and Thakur, SS, "A Controlled Variation Scheme in a Sequential Solver for Recirculating Flows. Part I. Theory and Formulation," Numerical Heat Transfer B-Fund. 25(3), 245 (1994).

37. Versteeg, HK, and Malalasekera, W, An Introduction to Computational Fluid Dynamics: The Finite Volume Method, Pearson Education Limited, England (1995).

${ }^{38 .}$ Miettinen, KM, Nonlinear Multiobjective Optimization. Kluwer:Boston, (1999).

39. Chankong, V, Haimes, YY, Multi-objective Decision Making Theory and Methodology. Elsevier Science, New York, (1983). 
${ }^{40 .}$ Mack, Y, Goel, T, Shyy, W, and Haftka, RT, "Surrogate Model Based Optimization Framework: A Case Study in Aerospace Design", accepted for publication in Evolutionary Computation in Dynamic and Uncertain Environments (eds. Yang S, Ong YS, Jin Y), Springer Kluwer Academic Press, (2005).

${ }^{41 .}$ Reboud, JL, Sauvage-Boutar, E, and Desclaux, J, "Partial Cavitation Model for Cryogenic Fluids," Cavitation and Multiphase Flow Forum, Toronto, Canada (1990).

42. Delannoy, Y, and Reboud, JL, "Heat and Mass Transfer on a Vapor Cavity," Proc. ASME Fluids Engineering Conference, Washington DC 165, 209 (1993).

${ }^{43 .}$ Deshpande, M, Feng, J, and Merkle, CL, "Numerical Modeling of the Thermodynamic Effects of Cavitation," Journal of Fluids Engineering 119, 420 (1997).

44. Tokumasu, T, Kamijo, K, and Matsumoto, Y, “A Numerical Study of Thermodynamic Effects of Sheet Cavitation," Proc. of ASME FEDSM'02, Montreal, Quebec, Canada (2002).

45. Tokumasu, T, Sekino, Y, and Kamijo, K, "A New Modeling of Sheet Cavitation Considering the Thermodynamic Effects," Proceedings of $5^{\text {th }}$ International Symposium on Cavitation, Osaka, Japan (2003).

${ }^{46 .}$ Hosangadi, A, Ahuja, V, and Ungewitter, RJ, "Generalized Numerical Framework for Cavitation in Inducers," ASME Paper FEDSM2003-45408 (2003).

47. Myers, RH and Montgomery, DC, Response Surface Methodology, John Wiley and Sons, Inc (1995).

48. Matheron, G, "Principles of Geostatistics", Economic Geology, 58:1246-1266 (1963).

49. Orr, MJL, Introduction to Radial Basis Function Networks, Center for Cognitive Science, Edinburg University, EH 9LW, Scotland, UK. http://www.anc.ed.ac.uk/ mjo/rbf.html, (1996).

50. Goel, T, Haftka, RT, Shyy, W, and Watson, LT, "Pointwise RMS Bias Error Estimates for Design of Experiments", 44 ${ }^{\text {th }}$ AIAA Aerospace Sciences Meeting and Exhibit, Jan 9-12, Reno NV, AIAA2006-0724, 2006.

${ }^{51 .}$ Lophaven, SN, Nielsen, HB, and Sondergaard, J, "DACE: A Matlab Kriging Toolbox", version 2.0, Information and Mathematical Modeling, Technical University of Denmark, 2002. 
Table 1 Summary of relevant numerical experimentation on cryogenic cavitation

Reference

Main Features

Reboud et al. ${ }^{41}$

Delannoy and Reboud ${ }^{42}$

a) Potential flow equations with semi-empirical formulation

b) Simplistic interfacial heat transfer equation (suitable only for sheet cavitation)

Deshpande et al. ${ }^{43}$

c) Energy equation not solved

a) Explicit interface tracking

b) Simplistic model for vapor flow inside cavity (suitable only for sheet cavitation)

Tokumasu et al. ${ }^{44,45}$

c) Energy equation solved only in the liquid region

a) Explicit interface tracking

b) Improved model for vapor flow inside the cavity (suitable only for sheet cavitation)

c) Energy equation solved only in the liquid region

Hosangadi and Ahuja ${ }^{25}$

Hosangadi et al. ${ }^{46}$

a) Solved energy equation in the entire domain with dynamic update of material properties

b) Inconsistency noted with experimental results

c) Details on numerical issues and the choice of cavitation

Utturkar et al. $^{15}$ model parameters not explicitly provided

a) Solved energy equation in the entire domain with dynamic update of material properties

b) Test results for different fluids and reference conditions were consistent with the experimental results

Table $2 L_{2}$ norm of pressure and temperature difference

\begin{tabular}{ccccc}
\hline \hline Case & $C_{\text {dest }}$ & $C_{\text {prod }}$ & $P_{\text {diff }}$ & $T_{\text {diff }}$ \\
\hline Original & 1.0 & 80.0 & 11.26 & 0.353 \\
Baseline & 0.68 & 54.4 & 3.212 & 0.447 \\
\hline \hline
\end{tabular}

Table 3 Ranges of variables for global sensitivity analyses. $C_{d e s t}$ is the model parameter associated with the evaporation source term, $t_{\infty}$ is the reference time scale used in cavitation, $\rho_{v}^{*}$ and $L^{*}$ are the multiplication factors of vapor density and latent heat obtained from NIST database ${ }^{22}$, respectively

\begin{tabular}{cccc}
\hline \hline Variable & Minimum & Baseline & Maximum \\
\hline$C_{\text {dest }}$ & 0.578 & 0.68 & 0.782 \\
$t_{\infty}$ & $2.23 \times 10^{-3}$ & $2.65 \times 10^{-3}$ & $3.06 \times 10^{-3}$ \\
$\rho_{v}^{*}$ & 0.90 & 1.0 & 1.10 \\
$L^{*}$ & 0.90 & 1.0 & 1.10 \\
\hline \hline
\end{tabular}


Table 4 Summary of surrogate approximations of RMS surface pressure coefficient $\left(C_{p R M S}\right)$ and temperature $\left(T_{R M S}\right)$. PRS: polynomial response surface, KRG: Kriging

\begin{tabular}{ccc}
\hline \hline & $C_{p R M S}$ & $T_{R M S}$ \\
\hline \# of Training Points & 49 & 49 \\
Minimum of data & 1.567 & 82.284 \\
Mean of data & 1.648 & 82.569 \\
Maximum of data & 1.743 & 82.862 \\
\# of coefficients (PRS) & 10 & 11 \\
$\mathrm{R}^{2}{ }_{\text {adj }}$ (PRS) & 0.993 & 0.992 \\
PRESS (PRS) & 0.004 & 0.018 \\
Maximum error (PRS) & 0.009 & 0.029 \\
RMS error (PRS) & 0.004 & 0.013 \\
Process variance (KRG) & $3.67 \mathrm{e}-5$ & $6.14 \mathrm{e}-4$ \\
PRESS (KRG) & 0.0048 & 0.0154 \\
\hline \hline
\end{tabular}

Table 5 Predictions at four test points using polynomial response surface (PRS) and Kriging (KRG)

\begin{tabular}{cccccccccc}
\hline \hline$C_{\text {dest }}$ & $t_{\infty}$ & $\boldsymbol{\rho}_{v}^{*}$ & $\boldsymbol{L}^{*}$ & $C_{p R M S}$ & $T_{R M S}$ & $\begin{array}{c}C_{p R M S} \\
\text { (PRS) }\end{array}$ & $\begin{array}{c}T_{R M S} \\
\text { (PRS) }\end{array}$ & $\begin{array}{c}C_{p R M S} \\
\text { (KRG) }\end{array}$ & $\begin{array}{c}T_{R M S} \\
\text { (KRG) }\end{array}$ \\
\hline 0.578 & $2.23 \mathrm{e}-3$ & 1.0 & 1.0 & 1.645 & 82.565 & 1.646 & 82.570 & 1.644 & 82.569 \\
0.782 & $2.23 \mathrm{e}-3$ & 1.0 & 1.0 & 1.720 & 82.336 & 1.720 & 82.335 & 1.719 & 82.343 \\
0.578 & $3.06 \mathrm{e}-3$ & 1.0 & 1.0 & 1.582 & 82.807 & 1.582 & 82.808 & 1.582 & 82.808 \\
0.782 & $3.06 \mathrm{e}-3$ & 1.0 & 1.0 & 1.642 & 82.575 & 1.643 & 82.574 & 1.642 & 82.578 \\
\hline \hline
\end{tabular}

Table 6 Correlation parameters $(\theta)$ associated with the Kriging approximation of the two response functions $C_{p R M S}$ and $T_{R M S}$

\begin{tabular}{ccc}
\hline \hline$\theta$ & $C_{p R M S}$ & $T_{R M S}$ \\
\hline$C_{\text {dest }}$ & 0.662 & 0.496 \\
$t_{\infty}$ & 0.590 & 0.582 \\
$\rho_{v}^{*}$ & 0.131 & 0.079 \\
$L^{*}$ & 0.013 & 0.017 \\
\hline \hline
\end{tabular}

Table 7 Minimum and maximum of $P_{\text {diff }}$ and $T_{\text {diff }}$ based on 55 sample points

\begin{tabular}{cccc}
\hline & Minimum & Mean & Maximum \\
\hline$P_{\text {diff }}$ & 1.655 & 4.271 & 9.106 \\
$T_{\text {diff }}$ & 0.341 & 0.453 & 0.709 \\
\hline \hline
\end{tabular}

Table 8 Weights associated with different surrogate models (55 points). PRS: polynomial response surface approximation, KRG: Kriging, and RBNN: radial basis neural network

\begin{tabular}{cccc}
\hline & $w_{P R S}$ & $w_{K R G}$ & $w_{R B N N}$ \\
\hline$P_{\text {diff }}$ & 0.32 & 0.59 & 0.094 \\
$T_{\text {diff }}$ & 0.24 & 0.64 & 0.12 \\
\hline \hline
\end{tabular}


Table 9 Quality of different surrogate models (55 points). PRS: polynomial response surface approximation, KRG: Kriging, and RBNN: radial basis neural network

\begin{tabular}{cccc}
\hline \hline Surrogate & Measure & $P_{\text {diff }}$ & $T_{\text {diff }}$ \\
\hline \multirow{3}{*}{ PRS } & $R_{\text {adj }}^{2}$ & 0.993 & 0.976 \\
& PRESS & 0.704 & 0.0387 \\
& Max Error & 0.424 & 0.0257 \\
KRG & Process variance & 0.427 & 0.00088 \\
& PRESS & 0.353 & 0.0128 \\
RBNN & PRESS & 2.51 & 0.079 \\
& Max Error & 0.021 & 0.00198 \\
WTA & PRESS & 0.666 & 0.0135 \\
& Max Error & 0.133 & 0.0063 \\
\hline \hline
\end{tabular}

Table 10 Ranges of variables in compromise region

\begin{tabular}{lll}
\hline \hline Variable & Minimum & Maximum \\
\hline$C_{\text {dest }}$ & 0.578 & 0.660 \\
$t_{\infty}$ & $2.23 \times 10^{-3}$ & $2.65 \times 10^{-3}$ \\
\hline \hline
\end{tabular}

Table 11 Range of $\boldsymbol{P}_{\text {diff }}$ and $\boldsymbol{T}_{\text {diff }}$ based on 30 sample points in compromise zone

\begin{tabular}{cccc}
\hline \hline & Minimum & Mean & Maximum \\
\hline$P_{\text {diff }}$ & 1.655 & 3.167 & 7.226 \\
$T_{\text {diff }}$ & 0.356 & 0.460 & 0.559 \\
\hline \hline
\end{tabular}

Table 12 Weights associated with different surrogate models (compromise zone). PRS: polynomial response surface approximation, KRG: Kriging, and RBNN: radial basis neural network

\begin{tabular}{cccc}
\hline \hline & $w_{P R S}$ & $w_{K R G}$ & $w_{R B N N}$ \\
\hline$P_{\text {diff }}$ & 0.374 & 0.224 & 0.402 \\
$T_{\text {diff }}$ & 0.311 & 0.577 & 0.112 \\
\hline
\end{tabular}

Table 13 Quality of different surrogate models in compromise zone (30 points)

\begin{tabular}{cccc}
\hline \hline Surrogate & Measure & $P_{\text {diff }}$ & $T_{\text {diff }}$ \\
\hline \multirow{3}{*}{ PRS } & $R_{\text {adj }}^{2}$ & 0.998 & 0.999 \\
& PRESS & 0.136 & 0.0033 \\
& Max Error & 0.119 & 0.0025 \\
KRG & Process variance & 1.523 & 0.00064 \\
& PRESS & 0.232 & 0.0017 \\
RBNN & PRESS & 0.126 & 0.0098 \\
& Max Error & 0.073 & 0.0078 \\
WTA & PRESS & 0.152 & 0.0025 \\
& Max Error & 0.062 & 0.0013 \\
\hline \hline
\end{tabular}


Table 14 Predicted and actual $P_{\text {diff }}$ and $T_{\text {diff }}$ at two POF data points

\begin{tabular}{cccccccc}
\hline \hline$C_{\text {dest }}$ & $t_{\infty}$ & & Simulation & PRS & KRG & RBNN & WTA \\
\hline 0.578 & $2.36 \mathrm{e}-3$ & $P_{\text {diff }}$ & 1.949 & 1.878 & 1.950 & 1.916 & 1.910 \\
& & $T_{\text {diff }}$ & 0.463 & 0.464 & 0.464 & 0.464 & 0.464 \\
0.612 & \multirow{2}{*}{$2.49 \mathrm{e}-3$} & $P_{\text {diff }}$ & 2.027 & 2.057 & 1.822 & 2.108 & 2.025 \\
& & $T_{\text {diff }}$ & 0.0 .462 & 0.462 & 0.463 & 0.462 & 0.463 \\
\hline \hline
\end{tabular}

Table 15 Improvements in the predictions with data sampling process

\begin{tabular}{ccccc}
\hline \hline & $C_{\text {dest }}$ & $t_{\infty}$ & $P_{\text {diff }}$ & $T_{\text {diff }}$ \\
\hline Baseline & 0.680 & $2.65 \times 10^{-3}$ & 3.212 & 0.447 \\
Dataset 1 & 0.680 & $2.77 \times 10^{-3}$ & 2.226 & 0.463 \\
Dataset 2 & 0.646 & $2.65 \times 10^{-3}$ & 1.960 & 0.466 \\
Optimal & 0.578 & $2.36 \times 10^{-3}$ & 1.949 & 0.463 \\
\hline \hline
\end{tabular}

Table 16 Flow cases chosen for the hydrofoil geometry

\begin{tabular}{ccccccc}
\hline Fluid & $\begin{array}{c}\text { Case } \\
\text { name }^{\mathbf{2 3}}\end{array}$ & $\begin{array}{c}\text { Inlet temperature } \\
T_{\infty}\end{array}$ & $\begin{array}{c}\text { Freestream } \\
\mathrm{Re}_{\infty}\end{array}$ & $\begin{array}{c}\text { Cavitation } \\
\text { No. }\left(\sigma_{\infty}\right)\end{array}$ & $\begin{array}{c}C_{\text {dest }} \\
\text { (baseline) }\end{array}$ & $\begin{array}{c}C_{\text {prod }} \\
\text { (baseline) }\end{array}$ \\
\hline Liq. $\mathrm{N}_{2}$ & $296 \mathrm{~B}$ & $88.54 \mathrm{~K}$ & $1.1 \times 10^{7}$ & 1.61 & 0.68 & 54.4 \\
Liq. $\mathrm{N}_{2}$ & $283 \mathrm{~B}$ & $77.65 \mathrm{~K}$ & $4.7 \times 10^{6}$ & 1.73 & 0.68 & 54.4 \\
Liq. $\mathrm{H}_{2}$ & $249 \mathrm{D}$ & $20.70 \mathrm{~K}$ & $2.0 \times 10^{7}$ & 1.57 & 0.82 & 54.4 \\
\hline \hline
\end{tabular}

Table 17 Optimal model parameters of present transport based cavitation model ${ }^{4}$

\begin{tabular}{ccc}
\hline \hline Fluid & $C_{\text {dest }}$ & $t_{\infty}$ \\
\hline Liquid N2 & 0.578 & $2.36 \times 10^{-3}$ \\
Liquid H2 & 0.697 & $9.68 \times 10^{-4}$ \\
\hline \hline
\end{tabular}



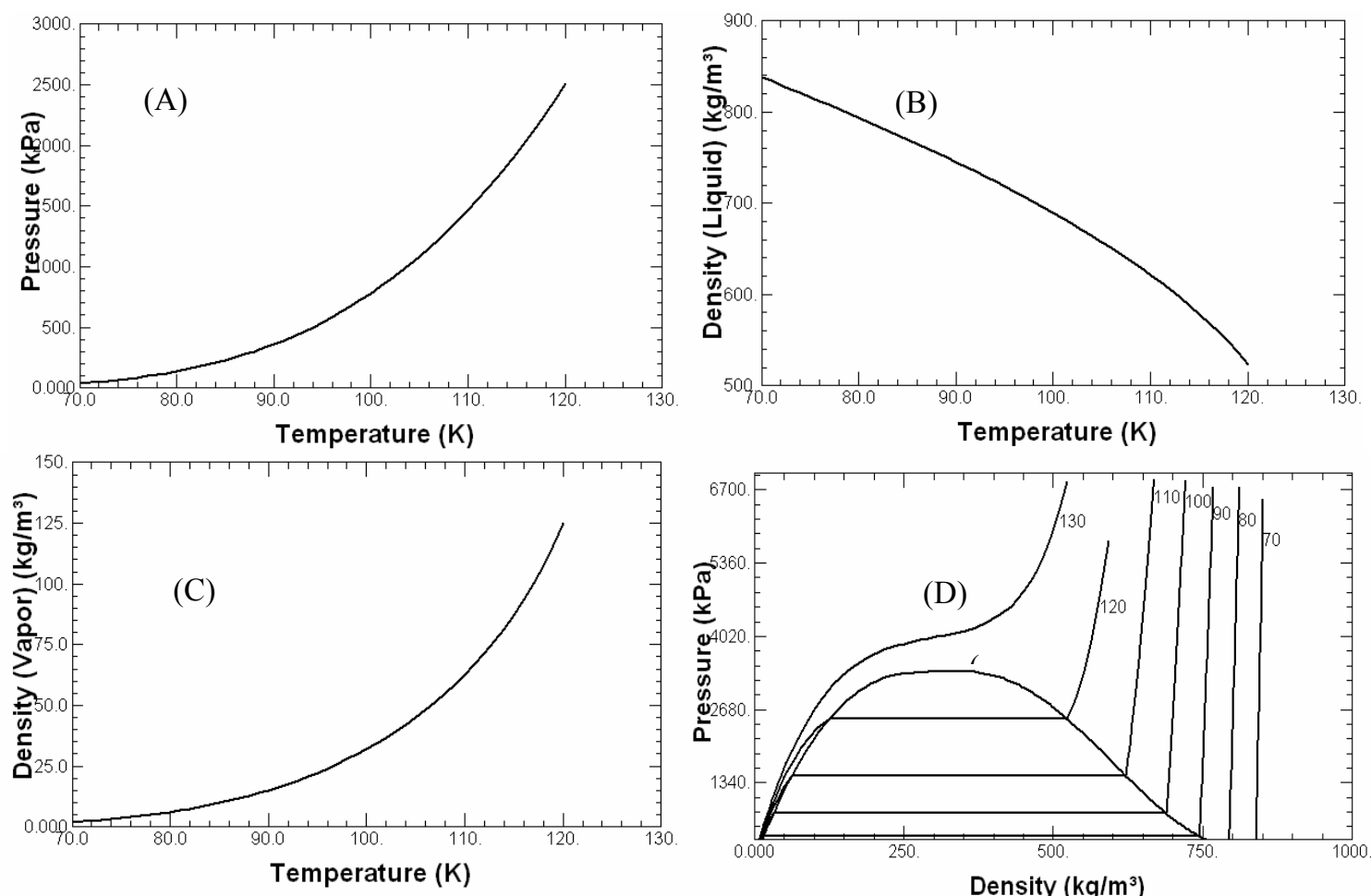

Figure 1 Variation of physical properties for liquid $\operatorname{Nitrogen}^{22}$ (A) vapor pressure v/s temperature along saturation line (B) liquid density $\mathbf{v} / \mathbf{s}$ temperature along saturation line (C) vapor density $\mathrm{v} / \mathrm{s}$ temperature along saturation line (D) Pressure-density chart -lines denote isotherms

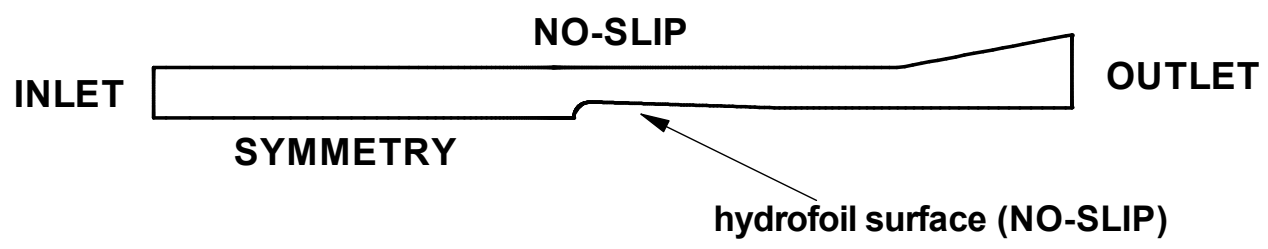

Figure 2 Illustration of the computational domain for the 2D hydrofoil ${ }^{23}$
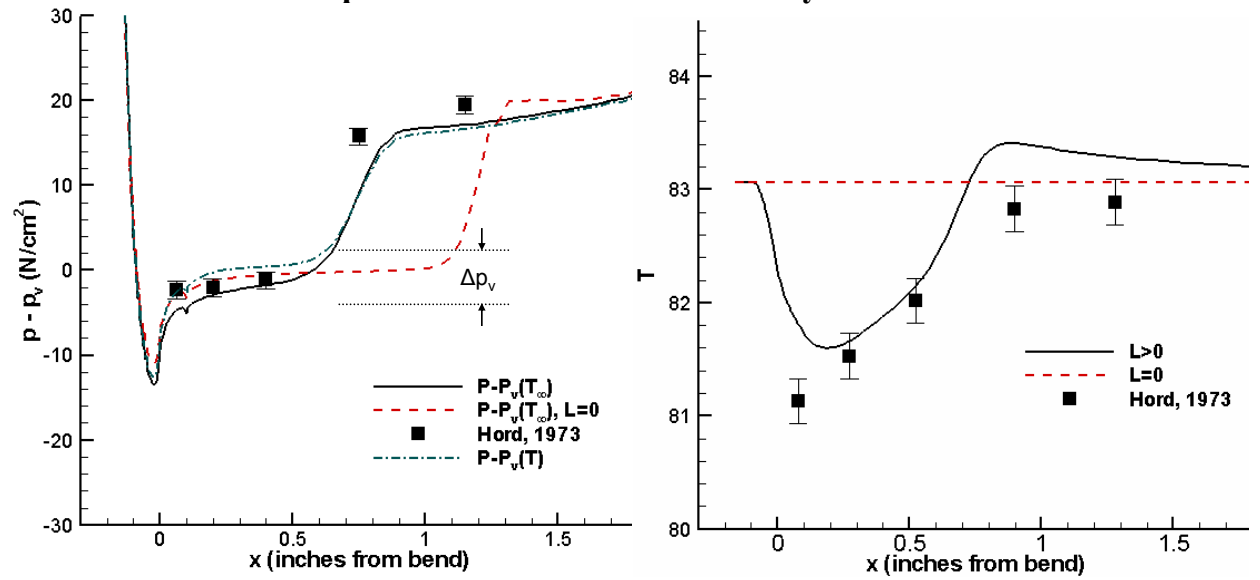

(A) Surface pressure

(B) Surface temperature

Figure 3 Surface pressure and temperature profile on 2D hydrofoil (Case $290 \mathrm{C}$, liquid $\mathrm{N}_{2}$ ) where the cavitation is controlled by (i) temperature-dependent vapor pressure (designated as $L>0$ ), and (ii) zero latent heat and hence isothermal flow field (designated as $L=0$ ). The range indicated by $\Delta p_{v}$ shows the level of variations in vapor pressure, $p_{v}$, caused by the temperature variations inside the cavity. (We use baseline parameters $C_{\text {dest }}=0.68, C_{\text {prod }}=54.4$ ) 

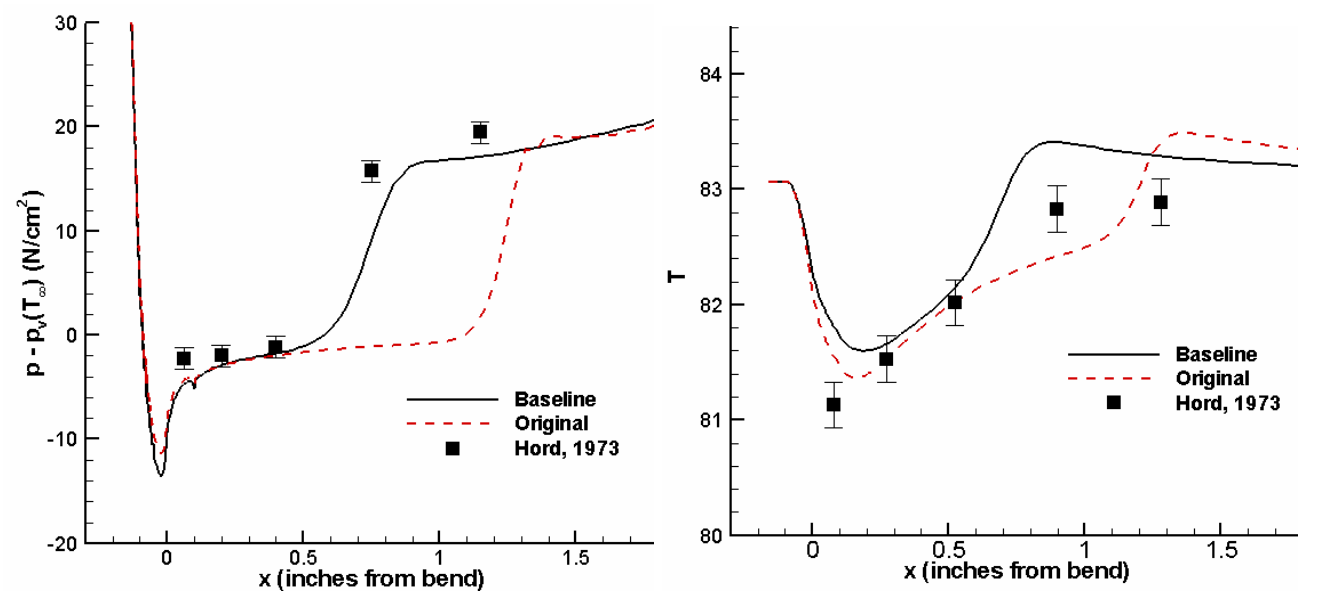

(A) Surface pressure

(B) Surface temperature

Figure 4 Effect of model parameters on surface pressure and temperature profile on 2D hydrofoil (Case 290C, liquid $\mathrm{N}_{2}$ ). Baseline parameters refer to $C_{\text {dest }}=\mathbf{0 . 6 8}, C_{\text {pred }}=\mathbf{5 4 . 4}$ (as suggested by Utturkar et al. ${ }^{15}$ ), and Original parameters refer to $C_{d e s t}=1.0$ and $C_{p r o d}=80.0$ (as specified by Merkle et al. ${ }^{4}$ ).

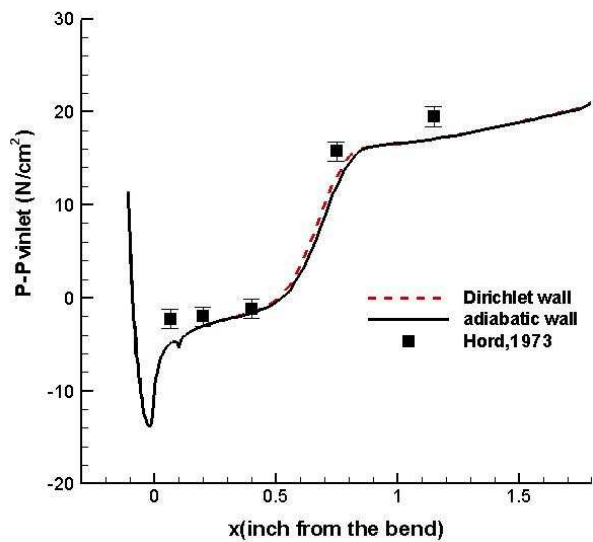

(A) Surface pressure

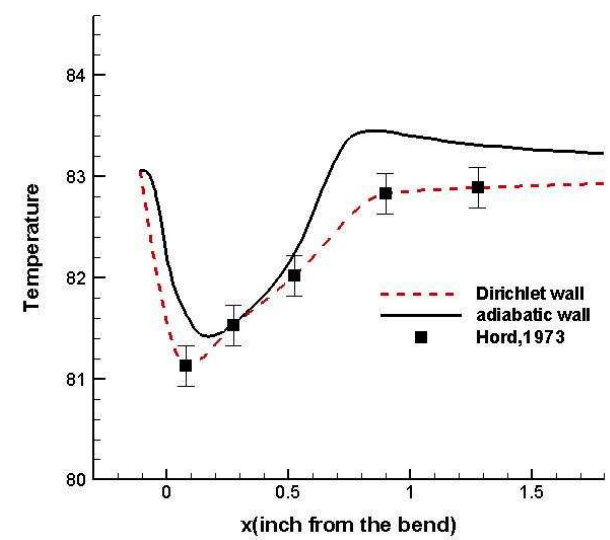

(B) Surface temperature

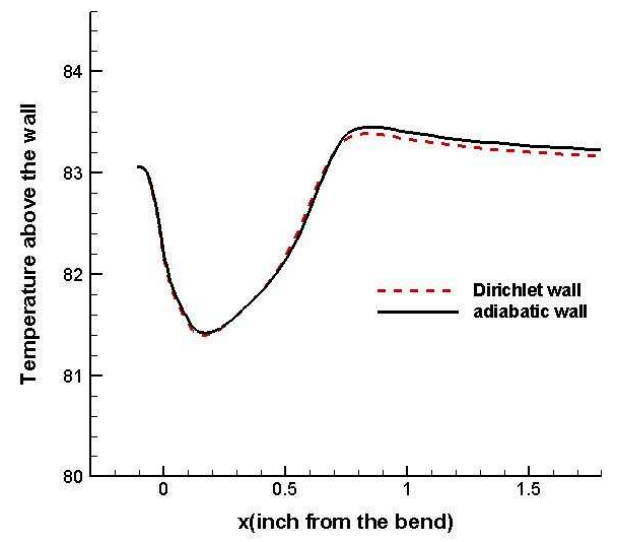

(C) Temperature on the first computational point

Figure 5 Impact of different boundary conditions on surface pressure and temperature profile on 2D hydrofoil (Case 290C, liquid $\mathrm{N}_{2}$ ) and predictions on first computational point next to boundary. 
GSA (Main effects, $C_{\mathrm{pRMs}}$ )
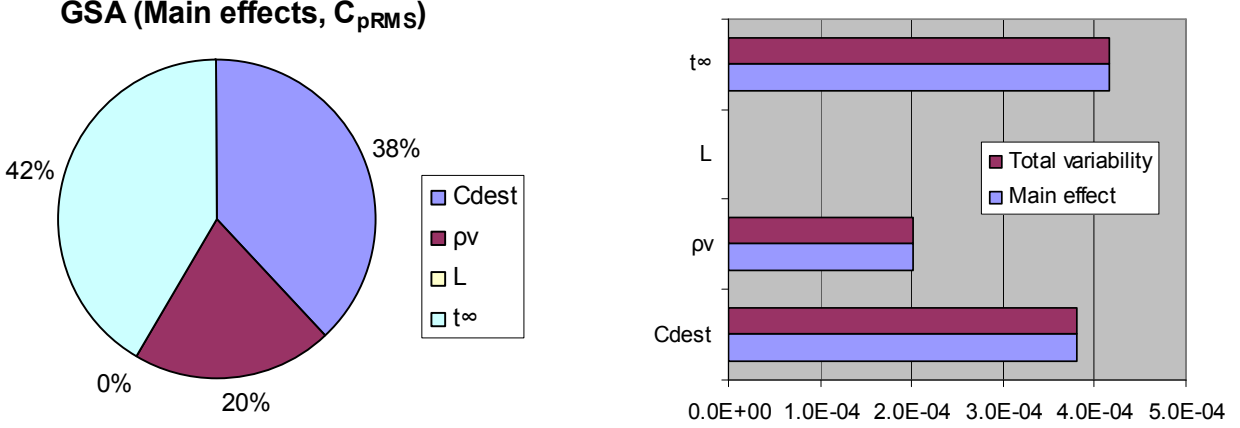

(A) Main and interaction effects (polynomial response surface approximations)

GSA (Main effects, $C_{\text {pRMs}}$ )
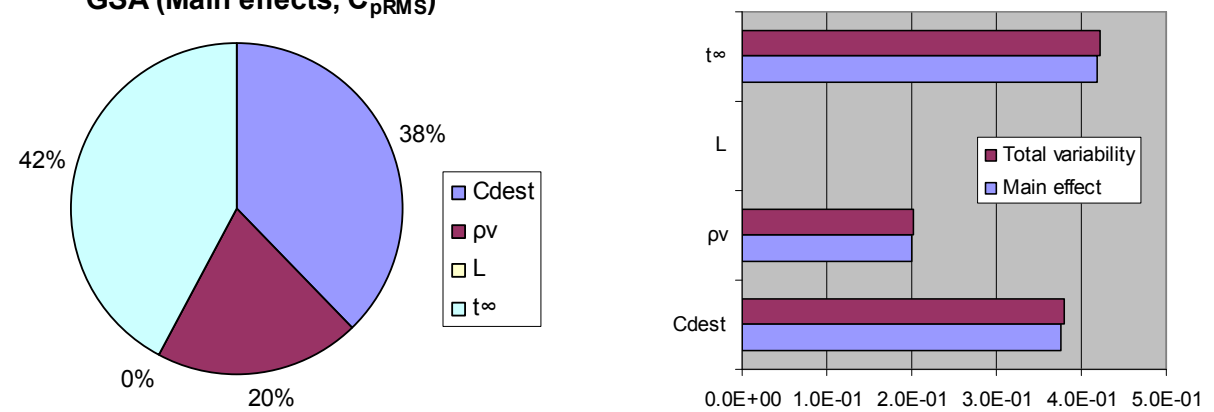

(B) Main and interaction effects (Kriging)

Figure 6 Main effects and interaction effects of different variables on the sensitivity of RMS surface pressure coefficient predictions
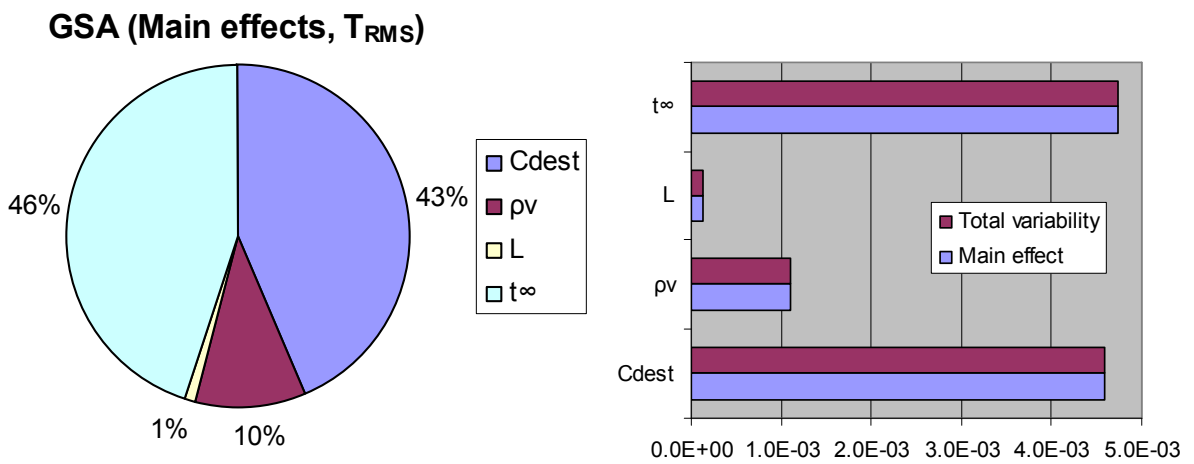

(A) Main and interaction effects (polynomial response surface approximations)

GSA (Main effects, $T_{\text {RMs }}$ )
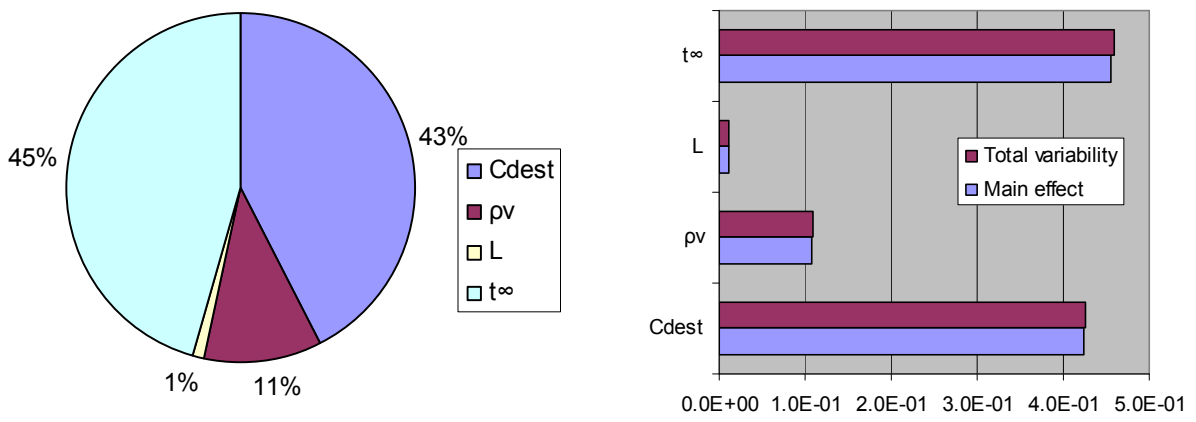

(B) Main and interaction effects (Kriging)

Figure 7 Main effects and interaction effects of different variables on the sensitivity of RMS surface temperature predictions 


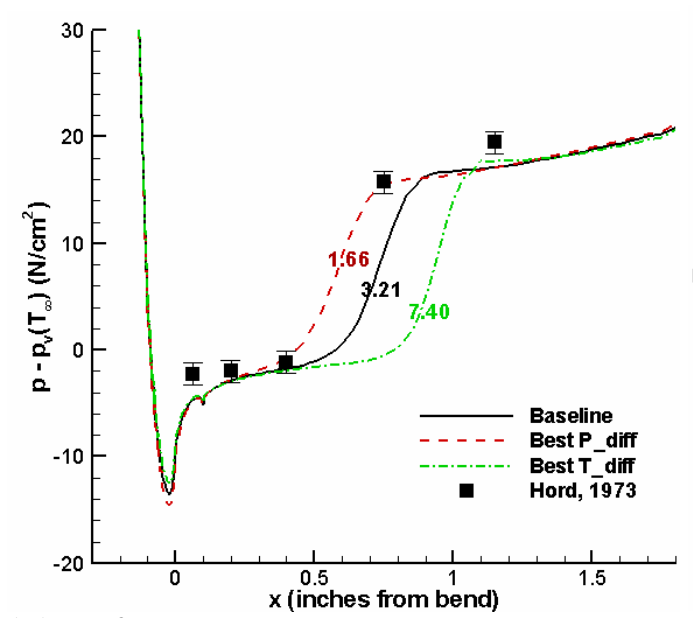

(A) Surface pressure

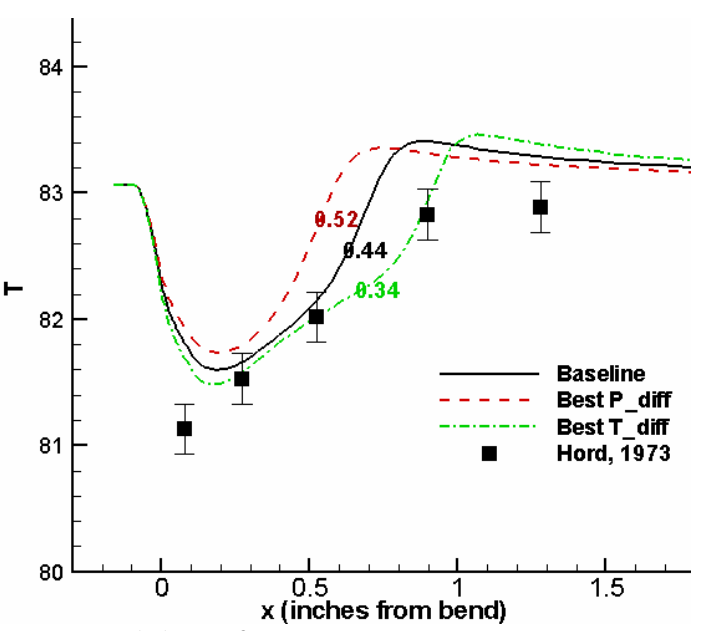

(B) Surface temperature

Figure 8 Surface pressure and temperature predictions using the model parameters that minimized $P_{\text {diff, }}, T_{\text {diff }}$ and baseline $\left(C_{\text {dest }}=0.68, C_{\text {pred }}=54.4\right)$ parameters. The number on each surface pressure or temperature profile represents $\boldsymbol{P}_{\text {diff }}$ or $\boldsymbol{T}_{\text {diff }}$ value associated with appropriate model parameters.

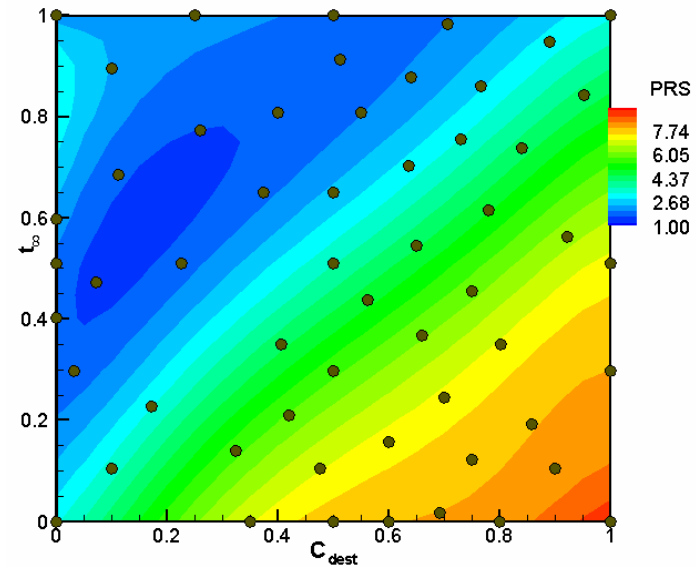

(A) Polynomial response surface

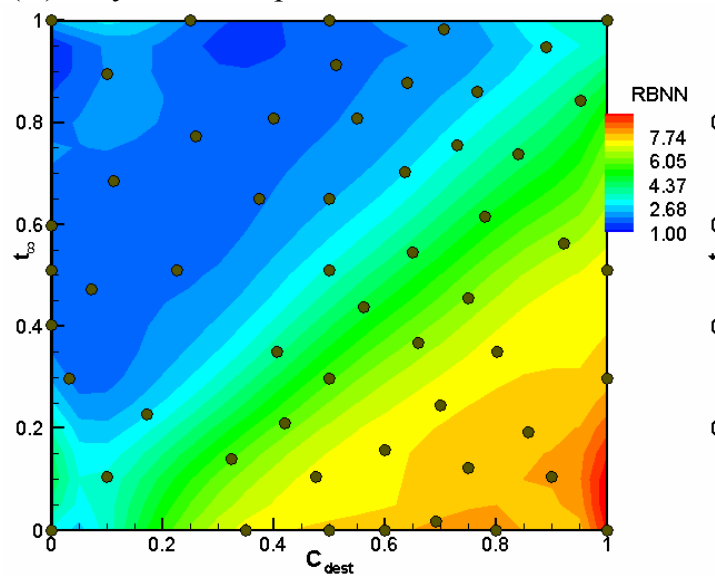

(C) Radial basis neural network

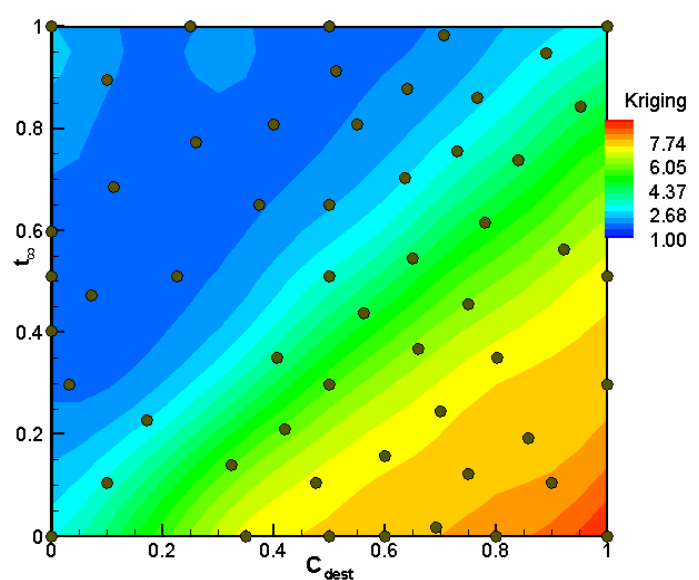

(B) Kriging

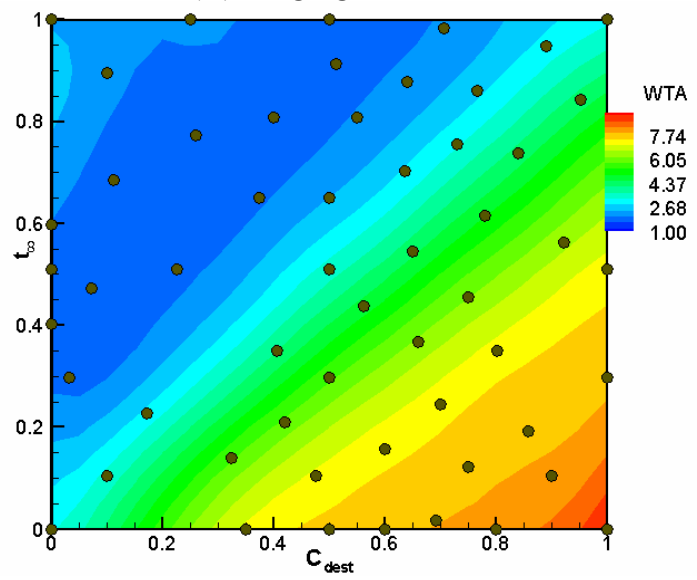

(D) Weighted average surrogate

Figure 9 Contours of pressure difference in normalized design space predicted using (A) PRS, (B) Kriging, (C) RBNN, and (D) WTA surrogate models 


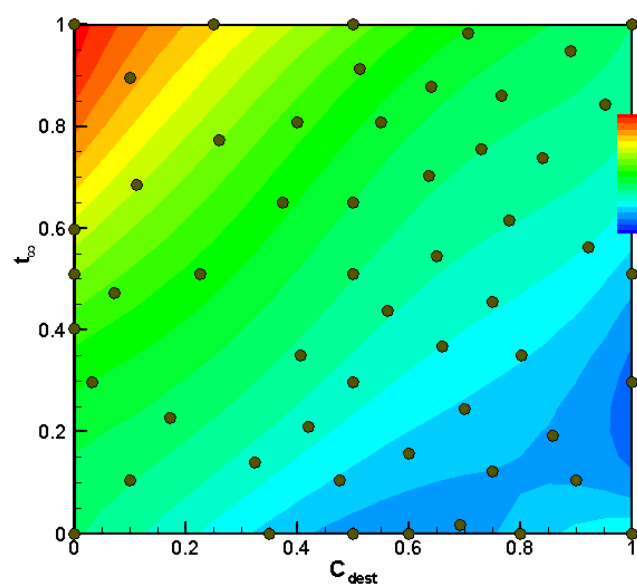

(A) Polynomial response surface

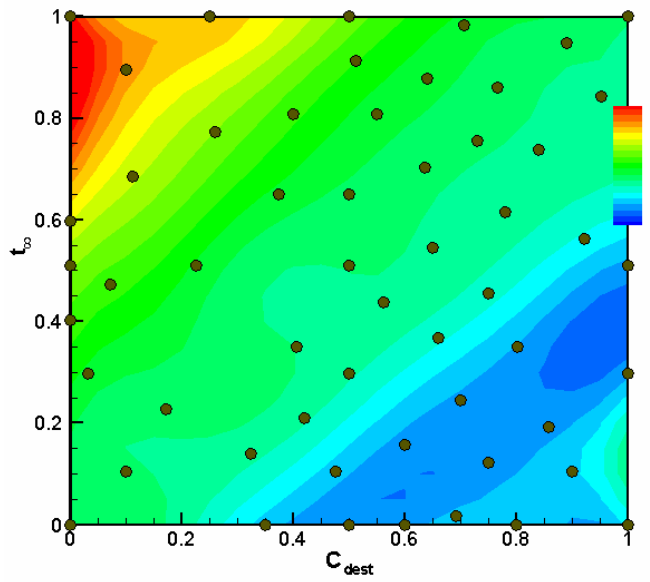

(C) Radial basis neural network

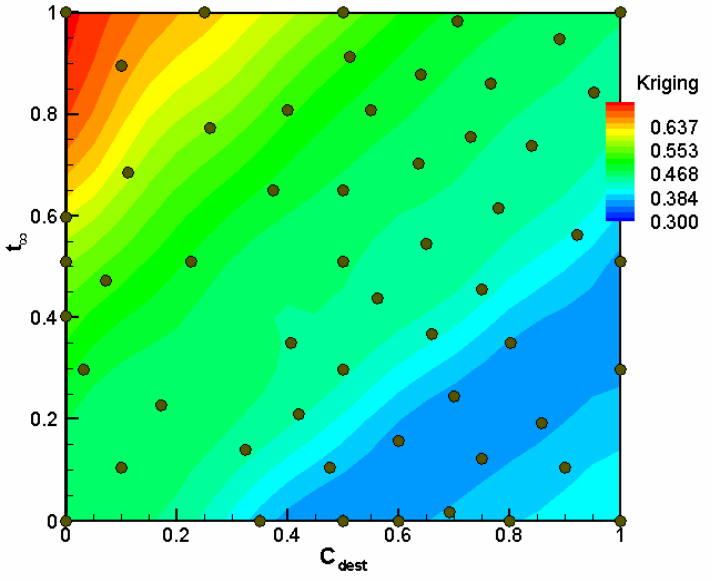

(B) Kriging

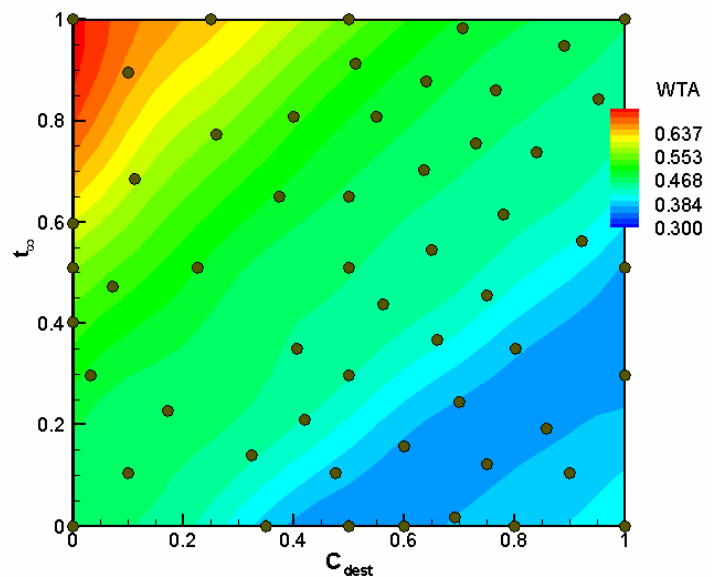

(D) Weighted average surrogate

Figure 10 Contours of temperature difference in normalized design space predicted using (A) PRS, (B) Kriging, (C) RBNN, and (D) WTA surrogate models

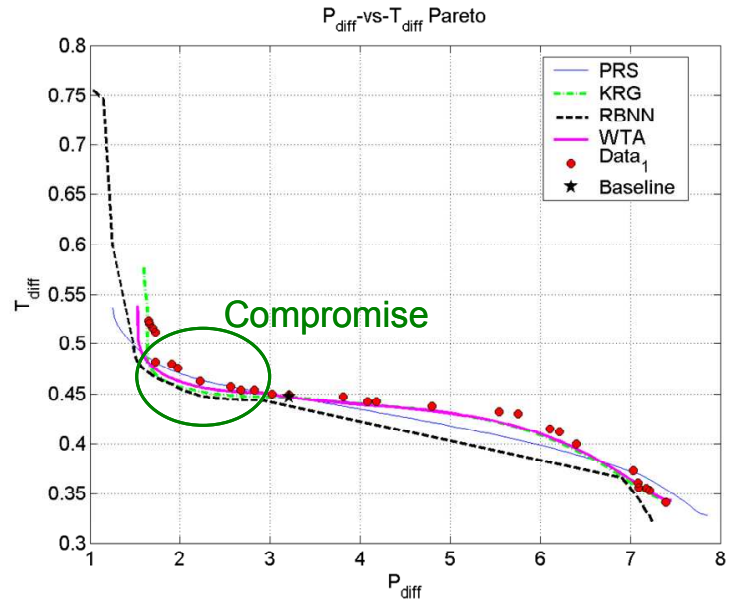

(A) Pareto optimal front in function space

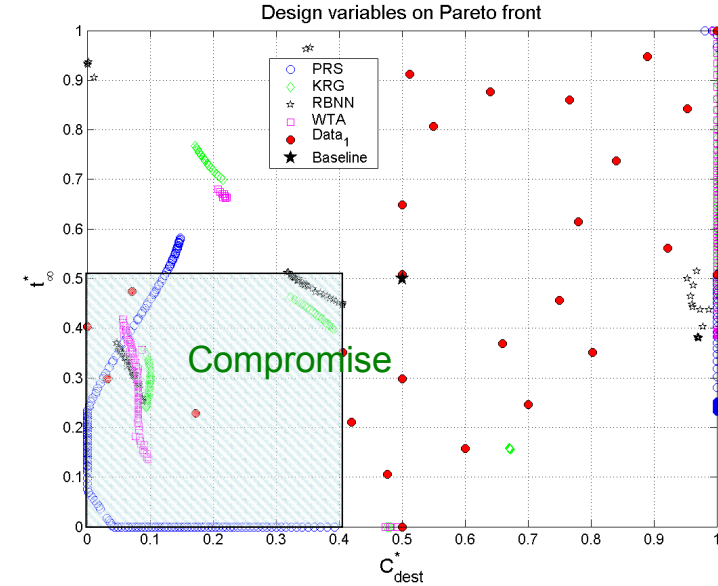

(B) Pareto optimal front in design space

Figure 11 Pareto optimal front (POF) and corresponding optimal points in (A) function space (B) normalized variable space (shaded region shows the compromise region) PRS: polynomial response surface, KRG: Kriging, RBNN: radial basis neural network, WTA: weighted average surrogate. 


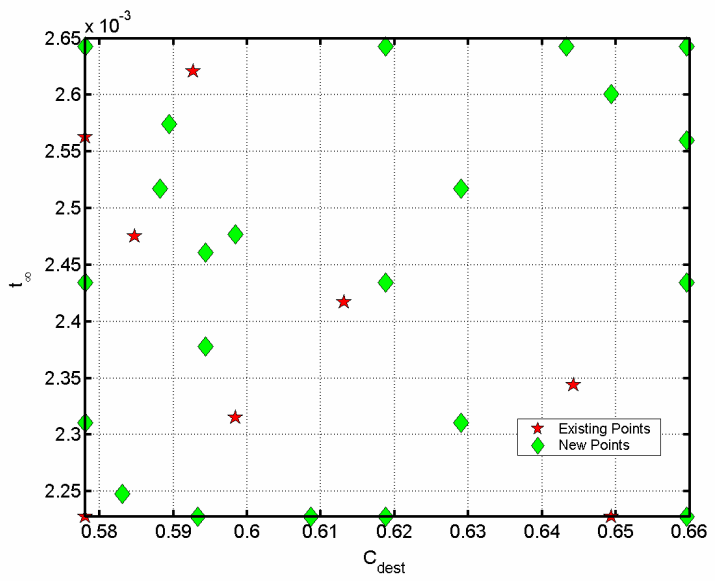

Figure 12 Existing and new sampled points in variables space

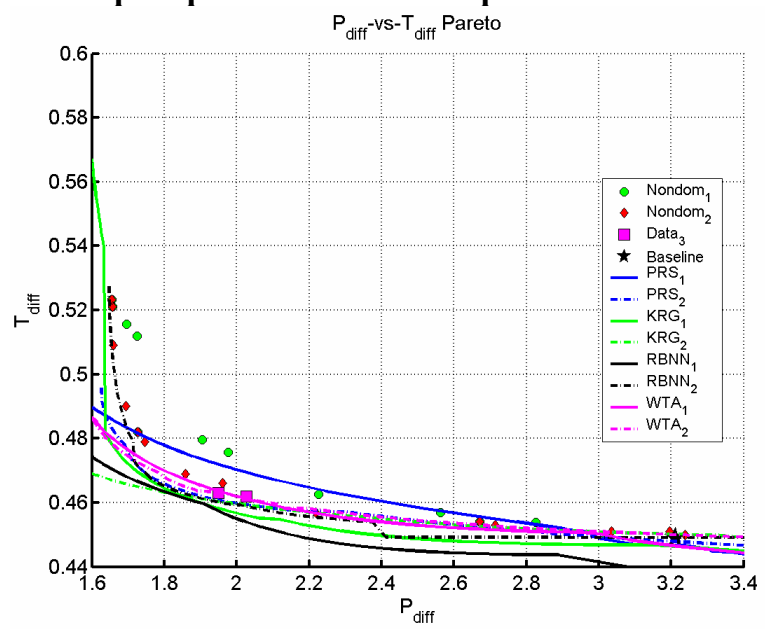

Figure 13 Pareto optimal front obtained using the surrogate models. Subscript 1 refers to data from complete design space (55 points) and Subscript 2 refers to the data in compromise zone. Range of POF is truncated to concentrate on the compromise solutions. PRS: polynomial response surface, KRG: Kriging, RBNN: radial basis neural network, WTA: weighted average surrogate.

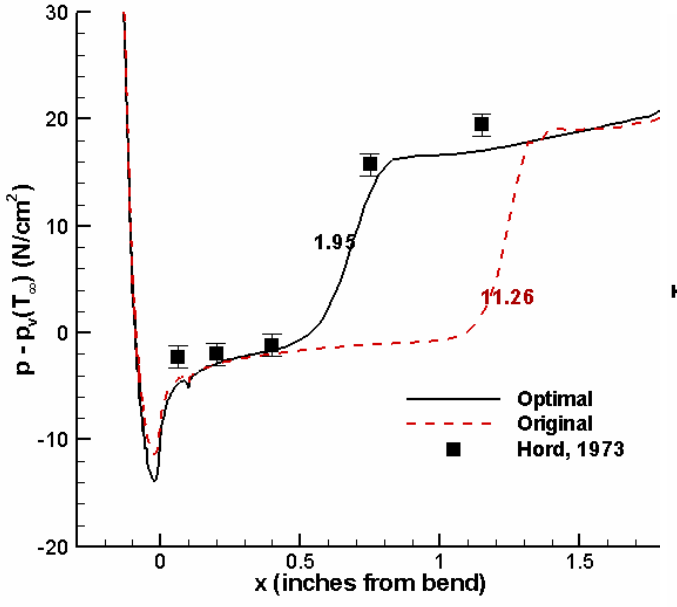

(A) Surface pressure

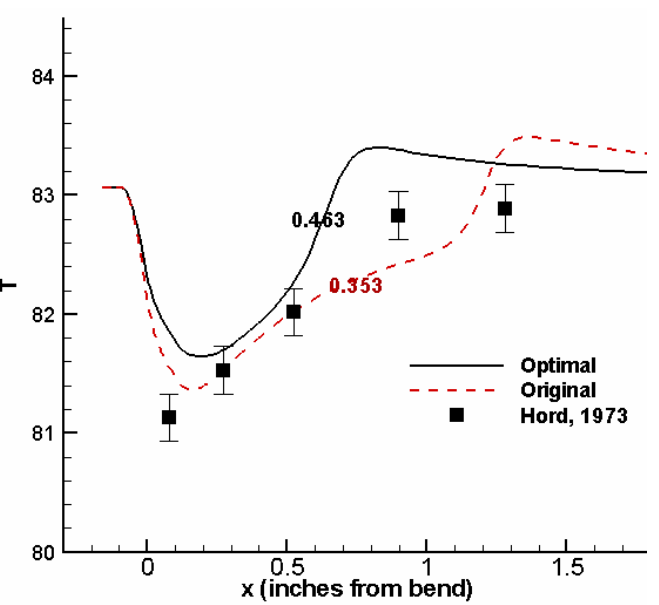

(B) Surface temperature

Figure 14 Surface pressure and temperature predictions using the model parameters corresponding to original, baseline and optimized values. The number on each surface pressure or temperature profile represents $\boldsymbol{P}_{\text {diff }}$ or $\boldsymbol{T}_{\text {diff }}$ value associated with appropriate model parameters. 


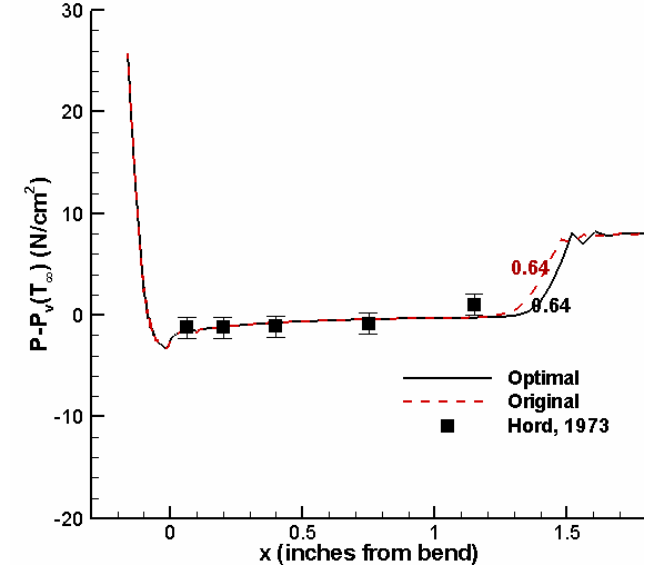

(A) Surface pressure (Case 283B)

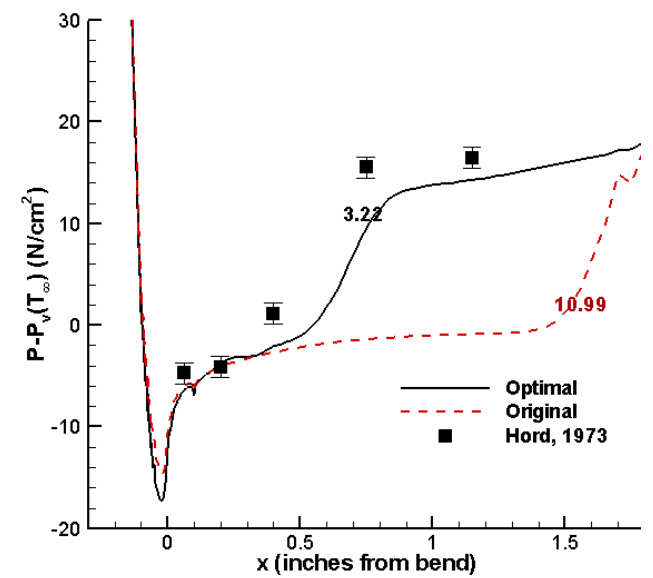

(A) Surface pressure (Case 296B)

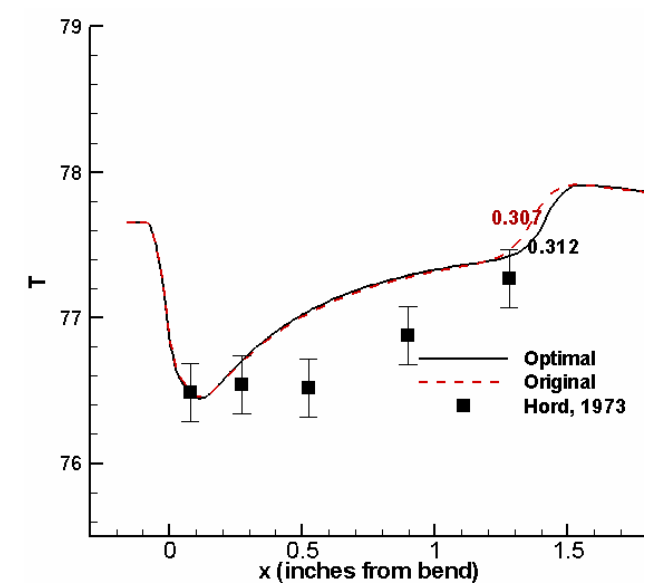

(B) Surface temperature (Case 283B)

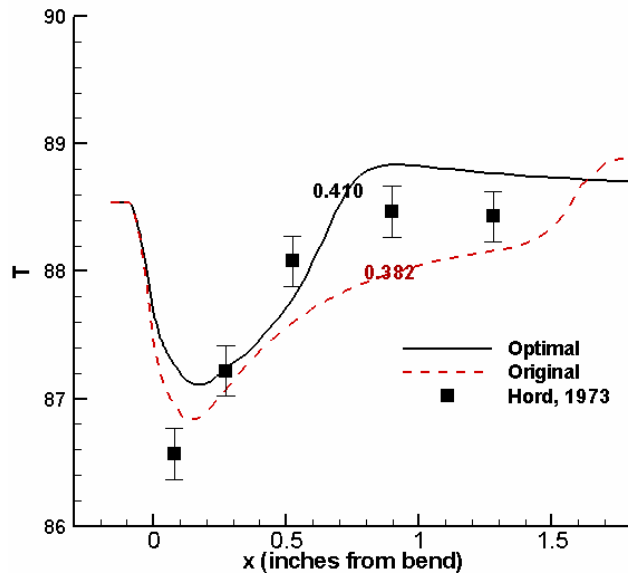

(B) Surface temperature (Case 296B)

Figure 15 Surface pressure and temperature predictions using the original parameters (liquid N2, $\left.C_{\text {dest }}=1.0, C_{\text {prod }}=\mathbf{8 0 . 0}, t_{\infty}=2.65 \times 10^{-3}\right)$, and optimized parameters $\left(C_{\text {dest }}=\mathbf{0 . 5 7 8}, C_{\text {pred }}=\mathbf{5 4 . 4}\right.$, $t_{\infty}=2.36 \times 10^{-3}$ ). The number on each surface pressure or temperature profile represents $\boldsymbol{P}_{\text {diff }}$ or $\boldsymbol{T}_{\text {diff }}$ value associated with appropriate model parameters.

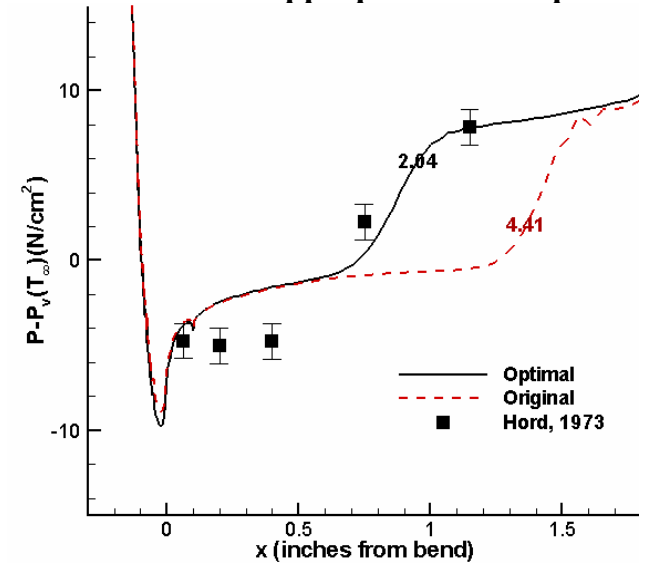

(A) Surface pressure

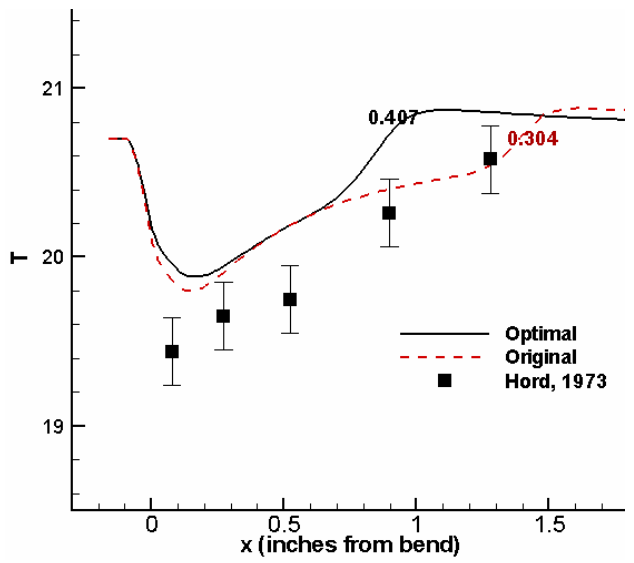

(B) Surface temperature

Figure 16 Surface pressure and temperature predictions (case 249D) using the original parameters (liquid H2, $\left.\boldsymbol{C}_{\text {dest }}=\mathbf{1 . 0}, \boldsymbol{C}_{\text {prod }}=\mathbf{8 0 . 0}, t_{\infty}=1.09 \times 10^{-3}\right)$, and optimized parameters $\left(\boldsymbol{C}_{\text {dest }}=\mathbf{0 . 6 9 7}, \boldsymbol{C}_{\text {pred }}=\right.$ 
54.4, $\left.t_{\infty}=9.68 \times 10^{-4}\right)$. The number on each surface pressure or temperature profile represents $\boldsymbol{P}_{\text {diff }}$ or $\boldsymbol{T}_{\text {diff }}$ value associated with appropriate model parameters.

\section{Appendix 1: Global Sensitivity Analysis (Sobol ${ }^{26}$, 1993)}

Global sensitivity analysis was first presented by $\mathrm{Sobol}^{26}$ in 1993. This method is used to estimate the effect of different variable on the total variability of the function. Some of the advantages of conducting a global sensitivity analysis include (i) assessing importance of the variables (ii) fixing non-essential variables (which do not affect the variability of the function) thus reducing the problem dimensionality etc. The theoretical formulation of the global sensitivity analysis is given as follows:

A surrogate model $f(\mathbf{x})$ of a square integrable objective as a function of a vector of independent input variables, $\mathrm{x}$ in domain $[0,1]$, is assumed and modeled as uniformly distributed random variables. The surrogate model can be decomposed as the sum of functions of increasing dimensionality as

$$
f(\mathbf{x})=f_{0}+\sum_{i} f_{i}\left(x_{i}\right)+\sum_{i<j} f_{i j}\left(x_{i}, x_{j}\right)+\cdots+f_{12 \ldots N}\left(x_{1}, x_{2}, \ldots, x_{N}\right)
$$

where $f_{0}=\int_{\mathbf{x}=0}^{1} f \mathrm{~d} \mathbf{x}$. If the following condition

$$
\int_{0}^{1} f_{i_{1} \ldots i_{s}} d x_{k}=0
$$

is imposed for $k=i_{1}, \ldots, i_{s}$, then the decomposition described in Equation (24) is unique. In context of global sensitivity analysis, the total variance denoted as $V(f)$ can be shown to be equal to

$$
V(f)=\sum_{i=1}^{N_{v}} V_{i}+\sum_{1 \leq i, j \leq N_{v}} V_{i j}+\ldots+V_{1 \ldots N_{v}}
$$

where $V(f)=E\left(\left(f-f_{0}\right)^{2}\right)$ and each of the terms in Equation (26) represents the partial contribution or partial variance of the independent variables $\left(V_{i}\right)$ or set of variables to the total variance and provides an indication of their relative importance. The partial variances can be calculated using the following expressions:

$$
\begin{aligned}
& V_{i}=V\left(E\left[f \mid x_{i}\right]\right) \\
& V_{i j}=V\left(E\left[f \mid x_{i}, x_{j}\right]\right)-V_{i}-V_{j} \\
& V_{i j k}=V\left(E\left[f \mid x_{i}, x_{j}, x_{j}\right]\right)-V_{i j}-V_{i k}-V_{j k}-V_{i}-V_{j}-V_{k}
\end{aligned}
$$

and so on, where $V$ and $E$ denote variance and expected value respectively. Note that $E\left[f \mid x_{i}\right]=\int_{0}^{1} f_{i} d x_{i}$ and $V\left(E\left[f \mid x_{i}\right]\right)=\int_{0}^{1} f_{i}^{2} d x_{i}$. Now, the sensitivity indices can be computed corresponding to the independent variables and set of variables. For example, the first and second order sensitivity indices can be computed as

$$
S_{i}=\frac{V_{i}}{V(f)}, \quad S_{i j}=\frac{V_{i j}}{V(f)}
$$

Under the independent model inputs assumption, the sum of all the sensitivity indices is equal to one.

The first order sensitivity index for a given variable represents the main effect of the variable but it does not take into account the effect of interaction of the variables. The total contribution of a variable on the total variance is given as the sum of all the interactions and the main effect of the variable. The total sensitivity index of a variable is then defined as: 


$$
S_{i}^{\text {total }}=\frac{V_{i}+\sum_{j, j \neq i} V_{i j}+\sum_{j, j \neq i} \sum_{k, k \neq i} V_{i j k}+\ldots}{V(f)}
$$

Note that the above referenced expressions can be easily evaluated using surrogate models of the objective functions. Sobol ${ }^{26}$ (1993) has proposed a variance-based non-parametric approach to estimate the global sensitivity for any combination of design variables using Monte Carlo methods. To calculate the total sensitivity of any design variable $x_{i}$, the design variable set is divided into two complementary subsets

of $x_{i}$ and $\mathrm{Z}\left(Z=x_{j}, \forall j=1, N_{v} ; j \neq i\right)$. The purpose of using these subsets is to isolate the influence of $x_{i}$ from the influence of the remaining design variables included in Z. The total sensitivity index for $x_{i}$ is then defined as

$$
S_{i}^{\text {total }}=V_{i}^{\text {total }} / V(f)
$$

where,

$$
V_{i}^{\text {total }}=V_{i}+V_{i, Z}
$$

$V_{i}$ is the partial variance of the objective with respect to $x_{i}$ and $V_{i, Z}$ is the measure of the objective variance that is dependent on interactions between $x_{i}$ and $Z$. Similarly the partial variance for $Z$ can be defined as $V_{z}$. Therefore the total objective variability can be written as

$$
V=V_{i}+V_{Z}+V_{i, Z}
$$

While Sobol ${ }^{26}$ had used Monte Carlo simulations to conduct the global sensitivity analysis, the expressions given above can be easily computed analytically once the response surface model is available.

\section{Appendix 2: Surrogate Modeling}

Surrogate models are developed as a computationally inexpensive method to evaluate design objectives. There are many surrogate models, e.g. polynomial response surface approximations, Kriging, radial basis neural network, support vector regression etc. A detailed discussion of different aspects of surrogate modeling was reviewed by Queipo et al. ${ }^{27}$ and $\mathrm{Li}$ and Padula ${ }^{28}$. We give a brief description of different surrogate models here.

\section{Polynomial Response Surface Approximation ${ }^{47}$}

The observed response $\mathrm{y}(\mathbf{x})$ of a function at point $\mathbf{x}$ is represented as a linear combination of basis functions $f_{i}(\mathbf{x})$ (mostly monomials are selected as basis functions) and coefficients $\beta_{i}$. Error in approximation $\varepsilon$ is assumed to be uncorrelated and normally distributed with zero mean and $\sigma^{2}$ variance. That is,

$$
y(\mathbf{x})=\sum_{i} \beta_{i} f_{i}(\mathbf{x})+\varepsilon \quad E(\varepsilon)=0, V(\varepsilon)=\sigma^{2}
$$

The polynomial response surface approximation of $y(\mathbf{x})$ is,

$$
\hat{y}(\mathbf{x})=\sum_{i} b_{i} f_{i}(\mathbf{x})
$$

where $b_{i}$ is the estimated value of the coefficient associated with the $i^{\text {th }}$ basis function $f_{i}(\mathbf{x})$. The coefficient vector $\mathbf{b}$ is obtained by minimizing the error in approximation $(e(\mathbf{x})=y(\mathbf{x})-\hat{y}(\mathbf{x}))$ at $N_{s}$ sampled design points in a least square sense as,

$$
\mathbf{b}=\left(X^{T} X\right)^{-1} X^{T} \mathbf{y}
$$

where $X$ is the matrix of basis functions and $\mathbf{y}$ is the vector of responses at $N_{s}$ design points. The quality of approximation is measures by computing the coefficient of multiple determination $R_{a d j}^{2}$ defined as, 


$$
\begin{aligned}
& R_{a d j}^{2}=1-\sigma_{a}^{2}\left(N_{s}-1\right) / \sum_{i=1}^{N_{s}}\left(y_{i}-\bar{y}\right)^{2} \\
& \text { where } \bar{y}=\sum_{i=1}^{N_{s}} y_{i} / N_{s} \text { and } \quad \text { adjusted RMS error at sampling points is given as }
\end{aligned}
$$

$\sigma_{a}=\sqrt{\sum_{i=1}^{N_{s}}\left(y\left(\mathbf{x}_{i}\right)-\hat{y}\left(\mathbf{x}_{i}\right)\right)^{2} /\left(N_{s}-N_{\beta}\right)} \quad$. For a good fit, $R_{a d j}^{2}$ should be close to 1. For more details on polynomial response surface approximation, refer to Myers and Montgomery ${ }^{47}$.

\section{Kriging $^{48}$}

Kriging is named after the pioneering work of D.G. Krige (a South African mining engineer). Kriging estimates the value of an objective function $y(\mathbf{x})$ at design point $\mathbf{x}$ as the sum of a linear polynomial trend model $\sum_{i=1}^{N_{v}} \beta_{i} f_{i}(\mathbf{x})$ and a systematic departure $Z(\mathbf{x})$ representing low (large scale) and high frequency (small scale) variations around the trend model.

$$
y(\mathbf{x})=\hat{y}(\mathbf{x})=\sum_{i} \beta_{i} f_{i}(\mathbf{x})+Z(\mathbf{x})
$$

The systematic departure components are assumed to be correlated as a function of distance between the locations under consideration. Gaussian function is the most commonly used correlation function.

$$
C(Z(\mathbf{x}), Z(\mathbf{s}), \boldsymbol{\theta})=\prod_{i=1}^{N_{v}} \exp \left(-\theta_{i}\left(x_{i}-s_{i}\right)^{2}\right)
$$

The parameters $\beta_{i}, \theta_{i}$ are obtained by minimizing the variance of the function.

\section{Radial Basis Neural Network ${ }^{49}$}

The objective function is approximated as a weighted combination of responses from radial basis functions (also known as neurons).

$$
\hat{y}(\mathbf{x})=\sum_{i=1}^{N_{R B F}} w_{i} a_{i}(\mathbf{x})
$$

where $a_{i}(\mathrm{x})$ is the response of the $i^{\text {th }}$ radial basis function at design point $\mathbf{x}$ and $w_{i}$ is the weight associated with $a_{i}(\mathbf{x})$. Mostly Gaussian function is used for radial basis function $a(\mathbf{x})$ as

$$
a=\operatorname{radbas}(\|\mathbf{s}-\mathbf{x}\| b) ; \operatorname{radbas}(n)=e^{-n^{2}}
$$

Parameter $b$ in the above equation is inversely related to a user defined parameter "spread constant" that controls the response of the radial basis function. A higher spread constant would cause the response of neurons to be very smooth and very high spread constant would result into a highly non-linear response function. Typically, spread constant is selected between zero and one. The number of radial basis functions (neurons) and associated weights are determined by satisfying the user defined error "goal" on the mean square error in approximation. Usual value of goal is the square of $5 \%$ of the mean response.

\section{Weighted Average Surrogate Model $^{29}$}

We develop a weighted average surrogate model as,

$$
\hat{y}_{w t a}(\mathbf{x})=\sum_{i}^{N_{S M}} w_{i}(\mathbf{x}) \hat{y}_{i}(\mathbf{x})
$$

where, $\hat{y}_{\text {wta }}(\mathbf{x})$ is the predicted response by the weighted average of surrogate models, $\hat{y}_{i}(\mathbf{x})$ is the predicted response by the $\mathrm{i}^{\text {th }}$ surrogate model and $w_{i}(\mathbf{x})$ is the weight associated with the $\mathrm{i}^{\text {th }}$ surrogate 
model at design point $\mathbf{x}$. Furthermore, the sum of the weights must be one $\left(\sum_{i=1}^{N_{S M}} w_{i}=1\right)$ so that if all the surrogates agree, $\hat{y}_{w t a}(\mathbf{x})$ will also be the same. Weights are determined as follows.

$$
\begin{aligned}
& w_{i}^{*}=\left(E_{i}+\alpha E_{\text {avg }}\right)^{\beta}, \quad w_{i}=w_{i}^{*} / \sum_{i} w_{i}^{*} \\
& E_{\text {avg }}=\sum_{i=1}^{N_{S M}} E_{i} / N_{S M} ; \quad \beta<0, \alpha<1
\end{aligned}
$$

where $E_{i}$ is the global data-based error measure for $i^{\text {th }}$ surrogate model. In this study, generalized mean square cross-validation error (GMSE) (leave-one-out cross validation or PRESS in polynomial response surface approximation terminology), defined in the Appendix 3, is used as global data-based error measure, by replacing $E_{i}$ by $\sqrt{G M S E_{i}}$. We use $\alpha=0.05$ and $\beta=-1$. The above mentioned formulation of weighting schemes is used with polynomial response surface approximation (PRS), Kriging (KRG) and radial basis neural networks (RBNN) such that,

$$
\hat{y}_{\text {wt.avg. }}=w_{\text {prs }} \hat{y}_{\text {prs }}+w_{k r g} \hat{y}_{k r g}+w_{r b n n} \hat{y}_{r b n n}
$$

\section{Appendix 3: Generalized Mean Square Cross-validation Error (GMSE or PRESS)}

In general, the data is divided into $k$ subsets ( $k$-fold cross-validation) of approximately equal size. A surrogate model is constructed $k$ times, each time leaving out one of the subsets from training, and using the omitted subset to compute the error measure of interest. The generalization error estimate is computed using the $k$ error measures obtained (e.g., average). If $k$ equals the sample size, this approach is called leave-one-out cross-validation (also known as PRESS in the polynomial response surface approximation terminology). Equation (44) represents a leave-one-out calculation when the generalization error is described by the mean square error (GMSE).

$$
G M S E=\frac{1}{k} \sum_{i=1}^{k}\left(y_{i}-\hat{y}_{i}^{(-i)}\right)^{2}
$$

where $\hat{y}_{i}^{(-i)}$ represents the prediction at $\mathbf{x}^{(i)}$ using the surrogate constructed using all sample points except $\left(\mathbf{x}^{(i)}, y_{i}\right)$. Analytical expressions are available for that case for the GMSE without actually performing the repeated construction of the surrogates for both polynomial response surface approximation (Myers and Montgomery, 1995, Section 2.7) and Kriging (Martin and Simpson, 2005) however here we used brute-force. The advantage of cross-validation is that it provides nearly unbiased estimate of the generalization error and the corresponding variance is reduced (when compared to split-sample) considering that every point gets to be in a test set once, and in a training set $k-1$ times (regardless of how the data is divided). 